\title{
Surface disentanglement and slip in a polymer melt: a molecular dynamics study
}

Article

Accepted Version

Kirk, J., Kroger, M. and Ilg, P. (2018) Surface disentanglement and slip in a polymer melt: a molecular dynamics study. Macromolecules, 51 (21). pp. 8996-9010. ISSN 0024-9297 doi: https://doi.org/10.1021/acs.macromol.8b01865 Available at https://centaur.reading.ac.uk/80127/

It is advisable to refer to the publisher's version if you intend to cite from the work. See Guidance on citing.

To link to this article DOI: http://dx.doi.org/10.1021/acs.macromol.8b01865

Publisher: American Chemical Society

All outputs in CentAUR are protected by Intellectual Property Rights law, including copyright law. Copyright and IPR is retained by the creators or other copyright holders. Terms and conditions for use of this material are defined in the End User Agreement.

\section{www.reading.ac.uk/centaur}

\section{CentAUR}

Central Archive at the University of Reading

Reading's research outputs online 


\title{
Surface disentanglement and slip in a polymer melt: A Molecular Dynamics study
}

\author{
J. Kirk, ${ }^{1}$ M. Kröger, ${ }^{2}$ and P. Ilg $^{1}$ \\ ${ }^{1}$ School of Mathematical, Physical and Computational Sciences, \\ University of Reading, Reading RG6 6AX, U.K. \\ ${ }_{2}^{2}$ Polymer Physics, Department of Materials, ETH Zurich, CH-8093 Zurich, Switzerland
}

(Dated: August 9, 2018)

We perform non-equilibrium molecular dynamics (MD) shear-flow simulations of an entangled polymer melt consisting of flexible linear chains. A steady-state rectilinear shear-flow is developed by sliding explicit walls with permanently grafted chains in a simple planar Couette flow geometry. As the channel average shear-rate is increased, a rapid coil-stretch transition of the surface endgrafted chains is observed. The corresponding primitive path network properties are investigated, revealing a disentanglement between surface grafted and non-grafted chains during the coil-stretch transition. Changes in slip length and surface friction are also measured. Grafted chains develop a trumpet like conformation at high shear-rates, which correlates with an increased relative density of entanglements near the free ends, a phenomenon that has already been considered by scaling models. The same mechanisms leading to slip in the current system may remain relevant for polymer melts of much higher (and more experimentally relevant) molecular weights. Therefore we use the simulation results to examine the predictions and assumptions of some existing theoretical models. The conclusions drawn from the simulation may be used in the future to further develop theoretical models for the surface rheology of polymer melts. 


\section{INTRODUCTION}

Flow instabilities are a serious problem for the plastic extrusion process. The reviews by Denn [1] and Hatzikiriakos [2] cover effects found in industry and experimental studies, including a summary of theoretical models. The 'stick-slip' instability, which is of interest in the present work, is one such example: for a variety of materials it has been found that, at a sufficiently high fixed pressure, the flow rate of the polymer in the die alternates between high and low rates, which are thought to correspond with slip and stick boundary conditions. If instead the die flow rate is held fixed then oscillations in the pressure occur [3]. These oscillations are further associated with varying degrees of 'die swelling', in which upon exiting the die the extrudate diameter increases. Oscillations in the extrudate diameter follow the pressure oscillations [3]. At sufficiently high flow rates this effect may lead to fracture of the extruded polymer melt.

In most extrusion setups the die channel dimension is much greater than the molecular dimension. Assuming that there is no bulk instability associated with boundary effects [4], in wide channels the importance of the surface is relegated to a boundary condition for the fluid velocity and molecular state. In the present work we investigate a non-equilibrium Molecular Dynamics (MD) simulation in the sliding plate geometry, also known as simple planar Couette flow.

The literature has primarily focused on two mechanisms that may explain macroscopic surface slip in entangled polymer melts. The first mechanism is chain desorption from the surface above a critical stress [5]. The second mechanism is flow induced surface disentanglement between adsorbed and non-adsorbed chains, thought to be the result of a coil-stretch transition of the adsorbed chains [6]. In addition the action of a low molecular weight species in a polydisperse sample containing a high molecular weight species has been investigated [7]. Here we focus on the phenomenon of shear-flow induced surface disentanglement. Although this work focuses on surface disentanglement, in general different mechanisms for slip may occur concurrently: e.g. desorption and disentanglement. However, advances in the functionalization of surfaces with grafted molecules [8-10] may mean that even simple models of permanently grafted chains have direct experimental applicability. Eliminating the desorption mechanism entirely may be desirable for certain applications, warranting more detailed investigation of this ideal case, for which much theoretical attention has focused. The system that we consider consists of chains that are permanently end-grafted (tethered) to the surfaces at regular positions. The grafting density is at the limit of the 'mushroom' regime whereby adjacent tethered chains only very weakly overlap.

This paper is organized as follows. In section [I] we survey the current state of the literature, focusing on theoretical models for the surface disentanglement. The aim of the present work is to critically examine some of the assumptions made in the theoretical models using state of the art MD simulations. In section 
III we introduce the MD model and methods. Z1 Primitive Path Analysis (PPA) [11-14] is employed to characterize the topological state of the fluid both at equilibrium and at high Weissenberg numbers. There is much evidence for a binary picture of entanglements [15-18]. In line with this picture, we are particularly interested in identifying the number and behavior of topological interactions between grafted (tethered - T) and non-grafted (free - F) chains away from equilibrium. We study the locations of topological interactions between different primitive paths, both in space and along the primitive path contours. We examine the correlations between primitive paths that are generated by the $\mathrm{Z} 1$ algorithm, the configurations of the corresponding bead-spring chains, and the rheological properties of the fluid. In section IV the main results are presented and discussed. In section $\mathrm{V}]$ final conclusions are made.

\section{SURVEY OF THEORETICAL DESCRIPTIONS OF SURFACE DISENTANGLEMENT AND SLIP IN POLYMER MELTS}

Theoretical scaling models describing the surface disentanglement were primarily developed by Brochard, de Gennes, and co-workers [19-22]. These models employ a picture whereby chains are permanently grafted by one end to the surface. Initially Brochard and de Gennes considered a simple model using the low grafting density limit whereupon a deformed grafted chain may increase the number of bulk chains it entangles with, and resultantly also increases the surface shear friction force, providing that the surfacenormal diameter, $D$, of the stretched 'cigar' conformation remains larger than a bulk entanglement 'tube' diameter: $D>a_{0}$. Then once $D<a_{0}$, the tethered chain experiences a complete disentanglement from the bulk chains. This leads to a sudden reduction of the shear friction force, and resultantly the strongly deformed chain relaxes rapidly and re-entangles with the bulk. The primary prediction was then of a 'marginal regime' where the stress remains constant for a range of applied shear-rates or slip velocities corresponding to $D \approx a_{0}$.

The first experimental observation of a marginal regime was made by Migler et al. [23], in a study in which the surface was specially treated to reduce the chain adsorption. Soon, further experiments exploring temperature [3] and molecular weight [6] dependence concluded that under many conditions the most important mechanism for a sudden onset of slip is disentanglement between adsorbed and non-adsorbed chains. For an accessible review of some seminal experiments investigating surface slip in polymer melts see Léger et al. [24].

Later scaling models were essentially more detailed variations of the original Brochard—de Gennes theory, aiming to describe several different observed scaling regimes. The higher grafting density cases were also considered [22]. Ajdari et al. [20] employed the analogy of a single chain with one end tethered to a 
nano-particle that is pulled through a melt. Contour length fluctuations [25] were assumed to aid relaxation of bond orientation far away from the tethered end, such that it was postulated that the deformation leads to a trumpet-shaped chain conformation for the tethered chain. Above a critical pull velocity, the narrow cylinder part of the trumpet crosses over to a diameter below the entanglement length. The contribution to the friction force acting on the fluid from this section of the chain contour is greatly reduced compared to the entangled section. Mhetar and Archer developed a similar scaling model based on the same ideas [26]. All of the scaling models are based upon a concurrent tethered chain alignment and consequent disentanglement between tethered and free chains. These models also assume that the bulk chains remain unperturbed from their equilibrium conformations: it has been demonstrated experimentally [27] that the onset of slip can occur at small Weissenberg numbers with respect to the bulk chain terminal relaxation time, such that the conformations of bulk chains are only weakly perturbed with respect to quiescence. Since many polymer melts shear-thin, any bulk deformation is expected to decrease the degree of slip following surface disentanglement compared to instances where a coil-stretch transition of surface chains occurs at a negligible bulk Weissenberg number.

Following the scaling models, adaptations of bulk tube models [28-30] were made, forming constitutive relations at the surface and in the bulk [31, 32]. In these models the surface constitutive relation is coupled to a bulk constitutive relation via stress balance and an additional diffusion of the tube contour due to convective constraint release [30] of the bulk chains. The tube model of Tchesnokov et al. [32] is based on the bulk GLaMM (Graham, Likhtman, Milner, McLeish) model [30]. In the surface region the shear stress is set equal to the product of an interfacial modulus $G_{I 0}$ with the mean tube segment shear orientation $S_{x y}^{I}$ of end-grafted chains: $\sigma_{x y}=G_{I 0} S_{x y}^{I}$. In their notation the interfacial modulus is $G_{I 0}=2 k_{\mathrm{B}} T \psi_{\mathrm{B} T}^{0} / a_{0}^{3}$, where $\psi_{\mathrm{B} T}^{0}$ is the volume fraction of entanglements that involve bulk and tethered chains which depends upon the grafting density within the model, $T$ is the temperature, $k_{\mathrm{B}}$ Boltzmann's constant, and $a_{0}$ is the mean spatial distance between entanglements, which is often considered equal to the equilibrium tube diameter. These models also considered a range of grafting densities from sparsely grafted 'mushroom' chains to the dense brush. The model of Tchesnokov et al. [32] was compared with experimental data which used a range of well controlled grafting densities [33]. As a comparison, Hoy and Grest [34] have examined entanglements between tethered and free species for a range of grafting densities above the Gaussian mushroom regime but below the dry brush regime, using MD simulations and PPA. Interestingly, although (i) the model of Tchesnokov predicts a sudden onset of slip, and (ii) the characteristic height of the surface region (found from the grafted chain surface normal height) is a quantity that is employed in the computation of the slip length, the key parameters controlling the effects of surface entanglements are independent upon the tube deformation: the surface modulus, $G_{I 0}$, and the fraction of tethered chain entanglements that are due to 
free chains $\left(\phi_{Z}\right.$ in [32]). The construction of the Joshi et al. [31] model is analogous in this respect. This means that these models imply the same assumption for the number of entanglements in tethered chains under strong shear as the one applied in the GLaMM model [30] for deformed bulk chains: the number of entanglements increases in proportion with the primitive path length of the chain (the tube diameter is assumed to be held fixed). Therefore, in the surface tube models, surface disentanglement is not explicitly modeled: these models assume that the coil-stretch transition precedes disentanglement. Instead, the initial slip is apparently due to the surface alignment of the tethered chains which is accelerated due to a limited convective constraint release mechanism [31] acting on the tethered chains from the bulk chains. It is also notable that the higher relaxation modes of the tethered chains, which do not depend on entanglements but become important once the chains are stretched, do not contribute to the stress in the interfacial tube models. However these models do include a contribution to the tube relaxation due to chain stretch via the tube retraction rate. In contrast the more detailed bulk GLaMM model does include the higher mode contributions to the stress.

Clearly it is desirable to examine the physical assumptions used in these various models. One limitation of experimental methods is that it is difficult to probe in detail what is happening on a molecular level at the surface, although recently Chennevire et al. [10] have been able to examine the change in the tethered chain monomer density profile following deformation, using a well defined end-grafted system. An alternative means of investigation is via computer simulation. In recent years simulation studies [35-39] have sought to examine in detail the molecular mechanisms which contribute to slip in entangled melts. A notable case is the work by Zhou et al. [37], where they found that adsorbed chains may slip across a smooth surface whilst remaining adsorbed, with the surface friction describing this process being dynamic: the total surface slip then depends upon a competition between disentanglement and this additional mechanism. Very recently Jeong et al. [38] have studied in more detail this phenomenology, identifying three flow regimes, universally valid at different molecular weights. For example, the fast flow regime is characterized by the tumbling motion of adsorbed chains which can lead to complete desorption. The majority of such simulation studies have used systems with partially adsorbing boundaries. One exception is a recent paper by Korolkovas et al. [39] that compared a coarse-grained simulation model of the collapse of permanently grafted brushes immersed in a dense polymer solution to corresponding experiments using two types of brushes. A degree of universality describing the brush collapse was found.

To isolate the coil-stretch phenomena from other mechanisms, and to examine the ideas and assumptions made in the various theoretical descriptions we here consider an idealized simulation consisting of chains that are permanently end-grafted to the surface, immersed in a melt of chains with the same molecular weight and chemistry. Although the scaling models are not quantitative, and the tube models are probably 
not applicable to the molecular weights we are able to simulate, we can examine some of the principles they are based on. In particular we consider some main questions:

(i) Is the general picture of the scaling models correct? I.e. is there a disentanglement associated with the coil-stretch transition?

(ii) Irrespective of the relationship between tethered-chain orientation and effective surface friction, do the scaling models accurately describe the evolution of the tethered-chain orientation with respect to shear-rate; are the stretched tethered conformations cigar-like or trumpet-like?

(iii) What is the correlation of any observed 'disentanglement' with surface friction and slip length?

In the supplementary material we also test the validity of the Stress Optical Rule (SOR) [40-42] at the surface in addition to a preliminary study into a basic assumption of both interfacial tube model formulations [31, 32]: that a SOR involving only the tethered chain species is valid.

\section{SIMULATION DETAILS AND EQUILIBRATION METHODS}

We use the confined Kremer-Grest (KG) bead-spring model [43] and parameters for flexible chains as in previous studies [14, 44], which is very similar to other models in the literature [45, 46]. The simulation comprises 676 linear chains, each consisting of $n=256$ Finitely Extensible Nonlinear Elastic (FENE) bonds, in the sliding plate geometry. The majority, 628 free chains, have two free ends. The top and bottom walls each carry 24 tethered chains, permanently adsorbed by one end to them, giving 48 such grafted chains in total. The grafting density of the chains is chosen as $\rho_{g}=0.00625 \sigma^{-2} \approx 2.8 \rho_{g}^{*}$, where we define the overlap grafting density $\rho_{g}^{*}=1 /\left\langle\boldsymbol{R}_{e, \text { bulk }}^{2}\right\rangle=0.0022 \sigma^{-2}$ for this chain length, where $\sigma$ is the LennardJones length, and $\boldsymbol{R}_{e, \text { bulk }}$ denotes the end-to-end vector of a $n$-chain in the bulk whose mean squared norm is actually very close to the one we find in the channel center. The same is true for all quantities to be discussed. For this reason we are going to label center channel quantities throughout by 'bulk'. LennardJones units time and mass we denote by $\tau_{\mathrm{LJ}}$ and $m_{b}$, respectively.

The grafted beads are located in a regular pattern using a two-dimensional slice of the Face Centered Cubic (FCC) lattice with miller indices (111), such that opposing surfaces have reflection symmetry. The grafting density is sufficiently low such that the chains are still in the 'Gaussian' (reflected random walk) regime; i.e. the grafted chains do not stretch significantly; therefore the grafting density that we investigate corresponds roughly with the recent experimental work of Chennevire et al. [10]. For this grafting density, we can use a previously establish protocol [44] to generate initial configurations of free and tethered chains: 
the free chains have a uniform start bead distribution everywhere in the channel; then, for both free and tethered chains, conformations are generated according to a fixed mean equilibrium bond length $(0.97 \sigma)$ and a bond angle distribution that is similar to the bulk equilibrium distribution, under the boundary constraint. The simulation box has dimensions $L_{x} \approx 4\left\langle\boldsymbol{R}_{e, \text { bulk }}^{2}\right\rangle^{1 / 2}, L_{y} \approx 3 L_{x} / 4$, and $L_{z} \approx L_{x} / 2$. The simulation box is bounded by reflective surfaces with normal unit vector $\hat{\boldsymbol{y}}$, so that the channel width is $L_{c}=L_{y}=$ $60.7 \sigma$. We use the Verlet algorithm [47] for integrating the equations of motion. For the Verlet algorithm, reflective surfaces are implemented in the following way: if a monomer crosses the surface at the current time then both the current and previous positions of the monomer are reflected in the surface plane. The unit vector coinciding with the surface velocity is $\hat{\boldsymbol{x}}$. It has been shown [48] that in the presence of surface grafted chains, many important equilibrium dynamic properties of a non-entangled melt weakly confined in a channel by two surfaces are close to invariant using a range of boundary potentials. It will be verified in section IV A that the center channel rheology of the entangled system we study is consistent with a corresponding simulation using periodic boundaries.

Some additional equilibration steps are necessary in this more entangled system, following the procedure of Cao and Likhtman [49]: initially we prepare a system that is half the final simulation size in the velocity direction with dimension $L_{x} \approx 2\left\langle\boldsymbol{R}_{e, \text { bulk }}^{2}\right\rangle^{1 / 2}$ while $L_{y}$ and $L_{z}$ remain unaltered. Initially we use soft non-bonded and harmonic bonded potentials which allow bond-crossing events. This set of soft interaction potentials has been termed 'soft MD' [50]. We have previously demonstrated that in the presence of a flat reflective surface the soft MD model has very similar static properties to the KG model [43]. Simulations using the soft MD model ran for several rotational relaxation times [40] of the tethered species, as determined from the longest relaxation mode from a spectrum fitted to the end-to-end vector correlation function. Following this the potentials were switched to the KG model using a 'push-off' over the first Lennard-Jones time, $\tau_{\mathrm{LJ}}$. The system then runs for a rotational relaxation time of the tethered species under the KG potential, $\tau_{\mathrm{T}} \approx 10^{6} \tau_{\mathrm{LJ}}$. Then every chain in the system is cloned; the cloned chains are displaced with respect to their parents by $L_{x} \hat{\boldsymbol{x}}$, and the box $\hat{\boldsymbol{x}}$ dimension is reset to $2 L_{x}$. Following this, the final double sized system runs for another $\tau_{\mathrm{T}}$ before any shear deformation is applied. In the non-equilibrium simulations the thermostat only acts in the neutral, $\hat{z}$, vorticity direction, and is applied uniformly across the channel. The thermostat friction is $\xi_{\mathrm{MD}}=0.5 m_{b} / \tau_{\mathrm{LJ}}$. For steady-state shear simulations, steady state observations begin $\tau_{\mathrm{T}}$ after the shear is switched on. It is validated that the transient stress following the stepped-shear has reached steady-state beyond the overshoot at this time, and that the primitive path data appears time-independent.

All computations were performed using a GPU 'device' except for on-the-fly data analysis performed on the CPU 'host'. The program was coded using Nvidia's CUDA platform [51]. For details of the core 


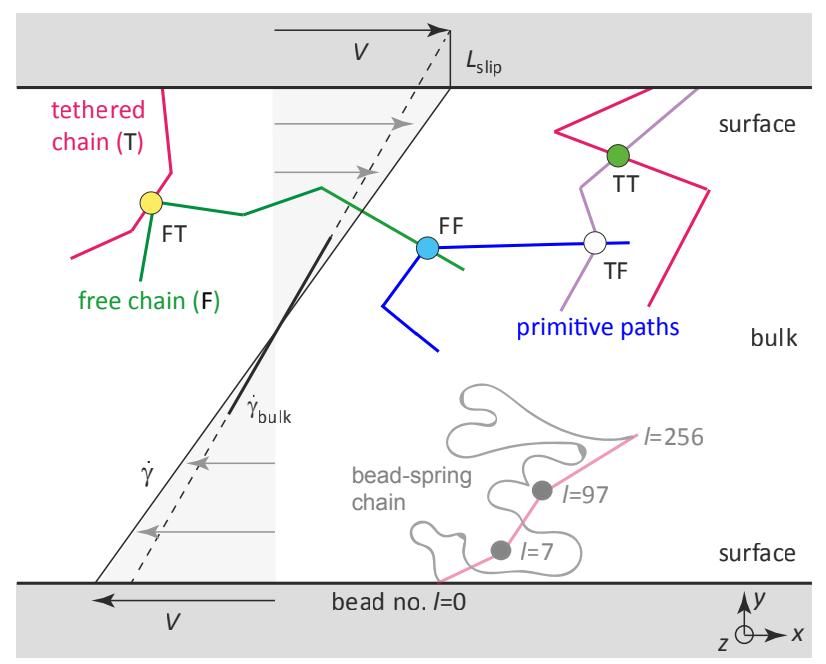

FIG. 1. Schematic of the simulation system. An example bead-spring chain conformation is represented by the wavy gray curve. Its primitive path is represented by the red lines that are connected at the locations of kinks in the primitive path (grey circles). Examples of the four possible types of kinks are represented:(a) FF: the cyan sphere represents a kink on a free chain primitive path that is $d u e$ to a free chain. (b) FT: the yellow sphere represents a kink on a free chain primitive path that is due to a tethered chain. (c) TT: the green sphere represents a kink on a tethered chain primitive path that is due to a tethered chain. (d) TF: the white sphere represents a kink on a tethered chain primitive path that is due to a free chain.

GPU MD code that we use see [52]. The code was extended for the present purpose to also allow for the simulation of non-periodic systems, including tethered chains and explicit surface deformation. Further technical details regarding the GPU code, including details of consistency checks with a corresponding $\mathrm{CPU}$ code, are given in the supplementary material.

Each steady state simulation ran for approximately six months, corresponding to approximately $10^{7} \tau_{\mathrm{LJ}}$ or $10 \tau_{\mathrm{T}}$ at an integration time step of $\Delta t=0.01 \tau_{\mathrm{LJ}}$ using a single $\mathrm{k} 10 \mathrm{GPU}$ and single CPU. The duration was sufficiently long to get small error bars for the static Z1 analysis, taken using many system snapshots. Error bars are generated under the assumption that snapshots separated by intervals of $\tau_{\mathrm{T}}$ are uncorrelated. For other dynamic observables it was not possible to construct error bars in this way since only a single observation was made over the entire simulation duration. An improvement, allowing for the construction of error bars, would have been to make, for example, ten separate observations each lasting $\approx \tau_{\mathrm{T}}$. However, the duration of observation was sufficiently long to give a clear qualitative picture of the shear-rate dependence of all observables investigated. 


\section{RESULTS AND DISCUSSION}

A schematic representation of the simulation system is given by Fig. 1. The fluid deformation is generated by displacing the grafted beads of the surface tethered chains. The opposing velocities at which grafted beads on the top and bottom walls are displaced are $V$ and $-V$ respectively. The speed of each wall is $V=\dot{\gamma} L_{c} / 2$, where $\dot{\gamma}$ is the channel average shear-rate, which from now on is referred to as the applied shear-rate. Due to the inhomogeneous nature of the fluid, an inhomogeneous shear-rate profile is developed such that in the center channel the 'bulk' shear-rate, $\dot{\gamma}_{\text {bulk }}$, is generally different from the applied shear-rate, $\dot{\gamma}$. We define $\dot{\gamma}_{\text {bulk }}$ from the gradient of a straight line fitted to the monomer velocity profile in the channel center.

Entanglements are frequently taken to be binary interactions between different chains [53]. We recognize that a kink in the final conformation of a primitive path is in some way dependent on all original positions of the monomers of all chains. However we may still identify the secondary primitive path whose final proximity directly led to the kink. This pairing introduces a binary picture of entanglements which in the following we exploit in order to examine primitive path interactions between different chain species (tethered $(\mathrm{T})$ and free $(\mathrm{F})$ ). Figure 1 illustrates the four possible types of binary interactions which lead to a kink on a primitive path that we say is due to the proximity of a different primitive path (self-entanglements are ignored by Z1). A kink on a tethered primitive path is due to the path meeting either another tethered path (TT kink) or a free path (TF kink). Likewise, a kink on a free primitive path is due to the path meeting either another free path (FF kink) or a tethered path (FT kink).

We define a free chain Weissenberg number using the bulk shear-rate: $\mathrm{Wi}_{\mathrm{F}}=\dot{\gamma}_{\text {bulk }} \tau_{\mathrm{F}}$, where $\tau_{\mathrm{F}}=$ $2.5 \times 10^{5} \tau_{\mathrm{LJ}}$ is the rotational relaxation time of the free chains $(n=256)$, which is defined from the longest Maxwell mode of a spectrum fitted to the end-to-end vector correlation function of free chains. The mean rotational relaxation time of all free chains in the channel is very similar to that of chains in the center of a channel without tethered chains [14], or that of a system of free chains using periodic boundaries. An alternative definition of the longest relaxation time, as used in previous work with which we may compare results [54], fits a single stretched exponential to the normalized end-to-end vector correlation function and then calculates the area under the curve. This integral is then equated with the relaxation time, which it coincides with exactly in the case of a single exponential. Both definitions give the same $\tau_{\mathrm{F}}$ within a relative error of $\approx 5 \%$ for this KG simulation. We also define a tethered chain Weissenberg number using the applied shear-rate: $\mathrm{Wi}_{\mathrm{T}}=\dot{\gamma} \tau_{\mathrm{T}}$. The rotational relaxation time of the tethered chains, $\tau_{\mathrm{T}}$, is related to $\tau_{\mathrm{F}}$ by $\tau_{\mathrm{T}} \approx 4 \tau_{\mathrm{F}}$ for this chain length, matching the ratio found using shorter completely non-entangled chains [44]. Table I gives the corresponding values of the shear-rates and Weissenberg numbers for each 


\begin{tabular}{cccc}
\hline \hline$\dot{\gamma} \tau_{\mathrm{LJ}}$ & $\mathrm{Wi}_{\mathrm{T}}$ & $\dot{\gamma}_{\text {bulk }} \tau_{\mathrm{LJ}}$ & $\mathrm{Wi}_{\mathrm{F}}$ \\
\hline $2.4 \times 10^{-6}$ & 2.4 & $2.64 \times 10^{-6}$ & 0.66 \\
$4.8 \times 10^{-6}$ & 4.8 & $4.56 \times 10^{-6}$ & 1.14 \\
$9.6 \times 10^{-6}$ & 9.6 & $7.20 \times 10^{-6}$ & 1.80 \\
$2.5 \times 10^{-5}$ & 25 & $1.45 \times 10^{-5}$ & 3.625 \\
$1 \times 10^{-4}$ & 100 & $6.50 \times 10^{-5}$ & 16.25 \\
$1 \times 10^{-3}$ & 1000 & $7.70 \times 10^{-4}$ & 192.5 \\
$1 \times 10^{-2}$ & 10000 & $8.50 \times 10^{-3}$ & 2125 \\
\hline \hline
\end{tabular}

TABLE I. Table giving the correspondence between the applied shear-rate, $\dot{\gamma}$, the tethered Weissenberg number, $\mathrm{Wi}_{\mathrm{T}}=\dot{\gamma} \tau_{\mathrm{T}}$, the bulk shear-rate, $\dot{\gamma}_{\text {bulk }}$, and the free Weissenberg number, $\mathrm{Wi}_{\mathrm{F}}=\dot{\gamma}_{\text {bulk }} \tau_{\mathrm{F}}$.

simulation. See Fig. 2 for an illustration of a small part of a primitive path network, including only those chains whose proximity results in a kink on the primitive path of a single selected (red) tethered chain $\left(\mathrm{Wi}_{\mathrm{T}}=9.6, \mathrm{Wi}_{\mathrm{F}}=1.8\right)$. In Fig. 2, the real space positions of the primitive path kinks are consistent with the areas of high overlap of the pervaded volumes of the chains involved in the kink.

\section{A. Center channel (bulk) rheology}

Here we investigate the center channel ('bulk') rheology. Figure 3a shows a snapshot of all primitive path conformations, folded in the simulation box at equilibrium. Tethered chain primitive paths are all red whereas the primitive paths of free chains are all blue. Cyan spheres represent FF kinks whereas yellow spheres represent FT kinks. TT and TF kinks are not represented. At equilibrium there is a small number of tethered primitive paths which reach the center channel. However once the free chains have become moderately oriented by the flow (e.g. at $\mathrm{Wi}_{\mathrm{F}}=16.25$ : see Fig. 3b) all tethered chains (primitive paths) are completely located immediately at the surfaces. Generally the center channel consists almost exclusively of free chains under all flow conditions.

The end-to-end unit vector of a chain is denoted by $\hat{\boldsymbol{k}}$. We define an orientation order parameter depending on the $\alpha$ component of $\hat{\boldsymbol{k}}$, averaged over chains of species $\mathrm{A}(\mathrm{A}=\mathrm{F}$ : free, or $\mathrm{A}=\mathrm{T}$ : tethered) with chain start bead at a distance $y$ from the surface as:

$$
S_{\alpha}^{\mathrm{A}}(y)=\left\langle P_{2}\left(\hat{k}_{\alpha}\right)\right\rangle_{y}^{\mathrm{A}}
$$

Here $P_{2}(x)=\left(3 x^{2}-1\right) / 2$ is the second Legendre polynomial. $S_{\alpha}^{\mathrm{A}}$ is bounded by 1 , corresponding to perfect alignment, whereas $S_{\alpha}^{\mathrm{A}}=0$ indicates isotropy. Figure 4 plots the orientation order parameter for the 


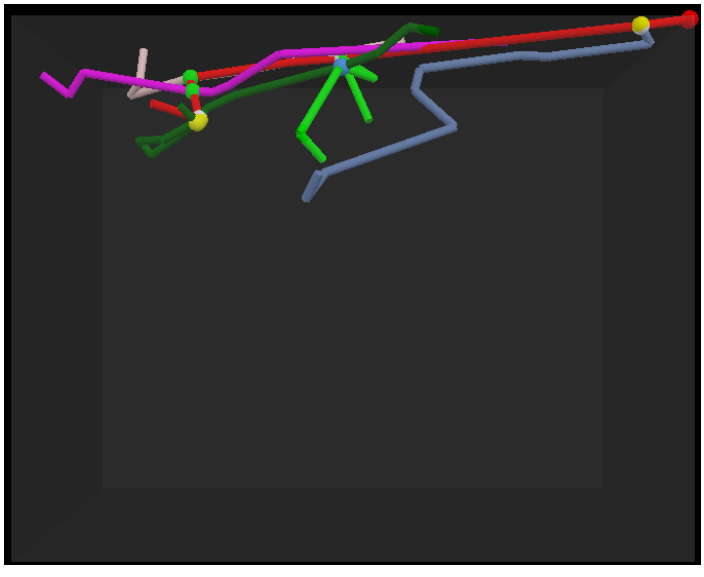

(a)

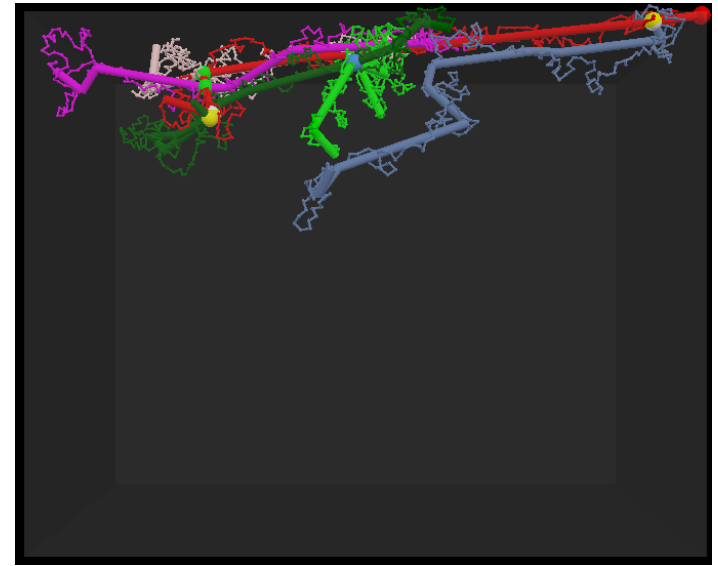

(b)

FIG. 2. (a) Unfolded primitive paths of all chains that are entangled with a single selected tethered chain (red), found using the $\mathrm{Z} 1$ algorithm. (b) Same as (a), decorated with the position of every bead in all of the chains prior to the Z1 algorithm. For illustrative purposes the primitive path conformations are placed inside a box with the same dimensions as the simulation box. There are two other tethered chains (beige and magenta) 'entangled' with the selected (red) chain. There are also three free chains (dark green, green, and blue) entangled with the selected chain. The red sphere represents the tethered end of the red chain/primitive path. Other spheres represent primitive path kinks which involve any two of the selected chains. The color code is consistent with other figures - TT: green sphere, TF: white sphere (hiding behind yellow spheres), FF: cyan sphere, FT: yellow sphere. Note that there is a topological interaction in this portion of the network that does not directly involve the red chain: indicated by the cyan sphere (FF kink). The snapshot is from a simulation using $\dot{\gamma}=9.6 \times 10^{-6} \tau_{\mathrm{LJ}}^{-1}\left(\mathrm{Wi}_{\mathrm{T}}=9.6, \mathrm{Wi}_{\mathrm{F}}=1.8\right)$.

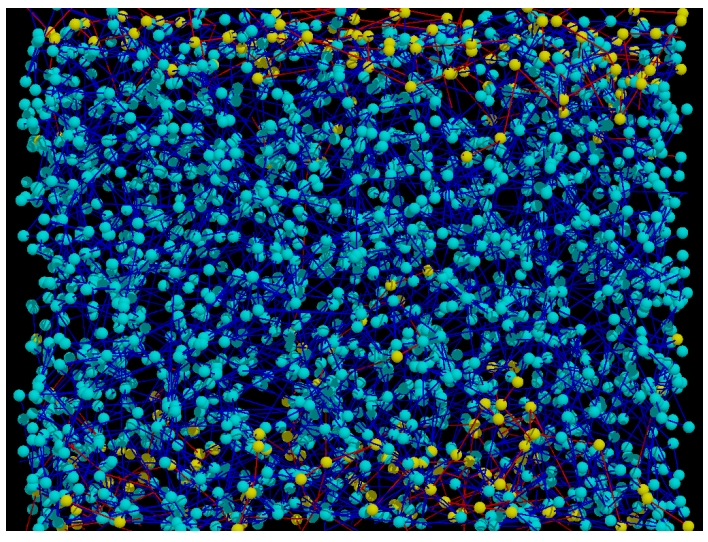

(a) Equilibrium

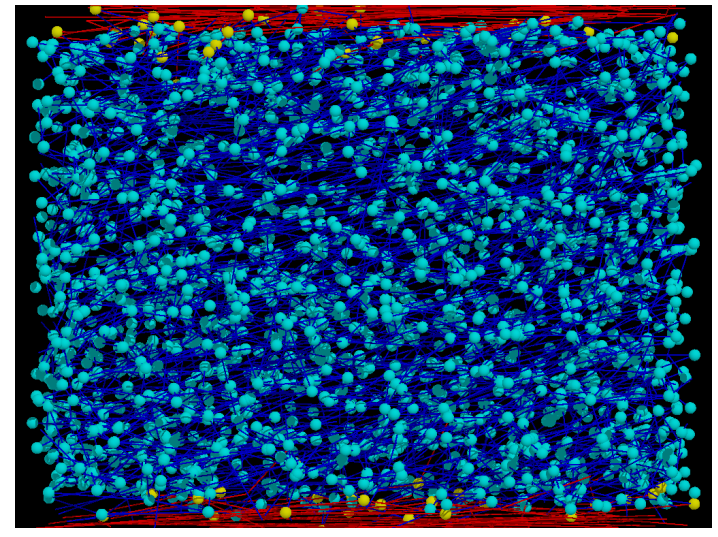

(b) $\mathrm{Wi}_{\mathrm{T}}=100, \mathrm{Wi}_{\mathrm{F}}=16.25$

FIG. 3. Snapshot of the complete set of folded primitive paths in the simulation box at (a) equilibrium; (b) $\dot{\gamma}=$ $10^{-4} \tau_{\mathrm{LJ}}^{-1}\left(\mathrm{Wi}_{\mathrm{T}}=100, \mathrm{Wi}_{\mathrm{F}}=16.25\right)$. Red lines: tethered chain primitive paths; blue lines: free chain primitive paths. Kinks in the primitive paths of free chains are indicated by spheres. Cyan spheres: kinks due to the proximity of another free chain (FF kinks); yellow spheres: kinks due to the proximity of a tethered chain (FT kinks). 


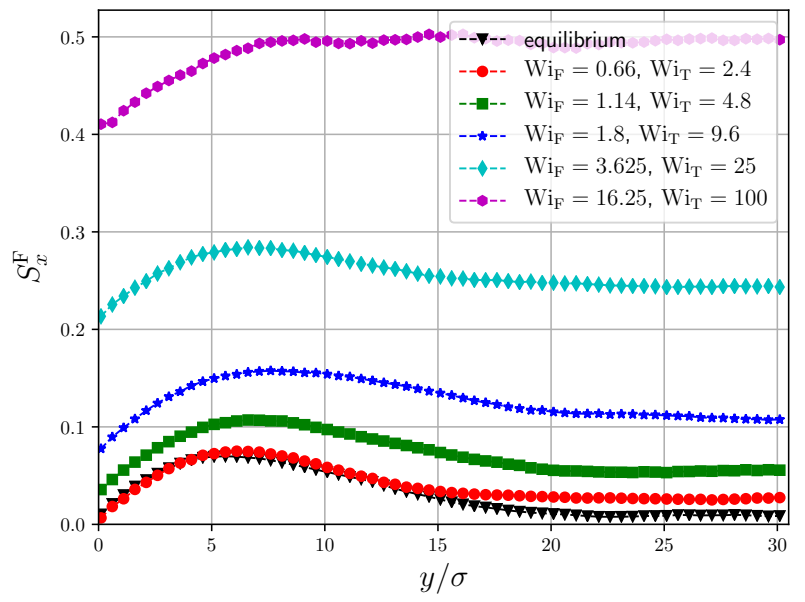

FIG. 4. Flow orientation order parameter of free chains, Eq. [1], as a function of the distance, $y \in\left[0, L_{c} / 2\right]$, from the position of the chain start (an end) bead to the nearest wall. Different plots correspond to different flow conditions (see the legend).

end-to-end unit vector of the free chains projected onto the flow direction, $S_{x}^{\mathrm{F}}(y)$, at different applied shearrates. Overall only mild spatial dependence of $S_{x}^{\mathrm{F}}(y)$ is observed; in equilibrium and at low shear rates there is a weak wall-induced orientation over a distance of approximately $\left\langle\boldsymbol{R}_{e, \text { bulk }}^{2}\right\rangle^{1 / 2}$. Figure 4 shows that the free chains only undergo moderate orientational ordering after the coil-stretch transition of tethered chains $\left(\mathrm{Wi}_{\mathrm{F}} \gtrsim 1.8, \mathrm{Wi}_{\mathrm{T}} \gtrsim 9.6\right.$ ) (see Fig. $11 \mathrm{a}$ for the orientation order parameter of tethered chains). Despite this, at $\mathrm{Wi}_{\mathrm{F}} \gtrsim 1$ there is already a non-negligible bulk shear-thinning which has an important influence on the degree of surface slip. The bulk shear-thinning acts against the effect of surface chain disentanglement with regard to surface slip. Figure 5a plots the bulk viscosity (defined from the shear-rate in the channel center as $\left.\eta_{\text {bulk }}=\sigma_{x y} / \dot{\gamma}_{\text {bulk }}\right)$ against the bulk shear-rate. The shear stress, $\sigma_{x y}$, is uniform across the channel and was found using the method of planes [55]. The monomeric velocity profiles are plotted in Fig. 6 .

Figure $5 \mathrm{a}$ is consistent with existing data for the non-linear rheology of the KG model under periodic boundary conditions - as a comparison see Fig. 1a of Kröger and Hess [56] (the density is set slightly lower - $0.84 \sigma^{-3}$ in the cited paper). Fitting a power-law relation $\eta_{\text {bulk }} \propto \dot{\gamma}_{\text {bulk }}^{-\mathcal{N}}$ using the largest four shear-rate data points available, determines an exponent $\mathcal{N}=0.61$, in close agreement with the earlier study [56] using a true bulk system. It is noted that for $\dot{\gamma}_{\text {bulk }} \gtrsim 0.01 \tau_{\text {LJ }}^{-1}$ the size of free chains in the flow direction can be greater than the box dimension which may lead to finite-size effects at such very fast deformation rates.

Next, we investigate 'entanglements' of free and tethered chains using the Z1 algorithm [11, 13] for the PPA analysis. The uppermost curve in Fig. 5 b plots the spatial density of kinks in the channel center (only 


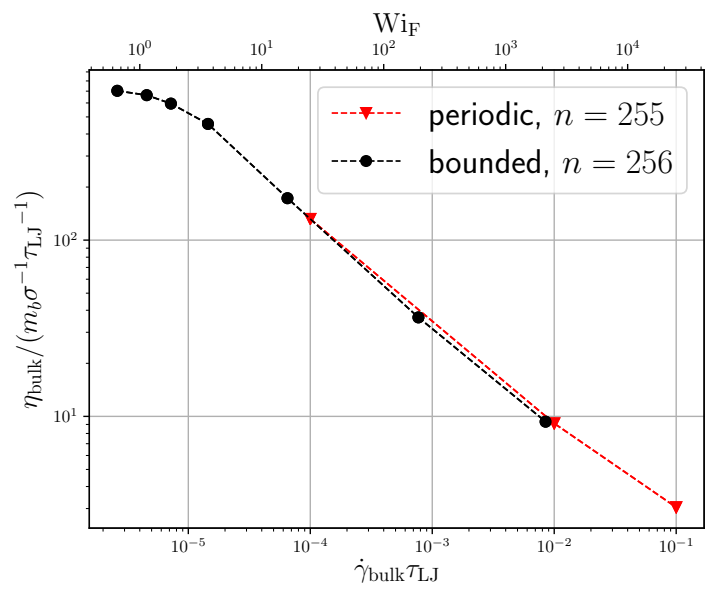

(a)

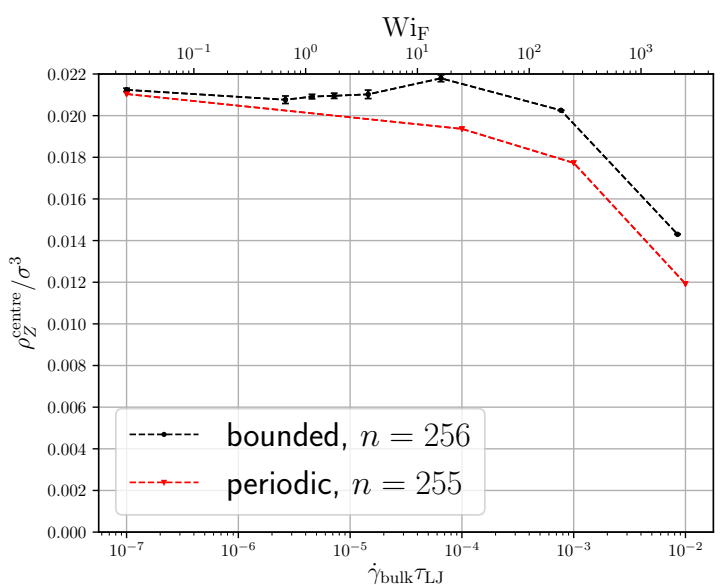

(b)

FIG. 5. (a) Channel center (black circles), and reference true bulk (red triangles), viscosity as a function of bulk shear-rate. Using the final four black points we determine the power law: $\eta_{\text {bulk }} \propto \dot{\gamma}_{\text {bulk }}^{-\mathcal{N}}$, with $\mathcal{N}=0.61$ compared to $\mathcal{N}=0.6$ in previous non-equilibrium MD investigations of the KG model using periodic boundaries [56]. The red data points from the periodic (true bulk) simulation use the SLLOD algorithm [57] and an almost identical molecular weight $(n=255)$. (b) Kink density in the channel center (black circles with error bars - kinks are at least $25 \sigma$ from the surface) and reference true bulk (red triangles) as a function of bulk shear-rate. Note that the density values at equilibrium are plotted in the figure against shear-rate: $\dot{\gamma}_{\text {bulk }}=10^{-7} \tau_{\mathrm{LJ}}^{-1}$. [\$ Can you make the red triangles larger in (b)?]

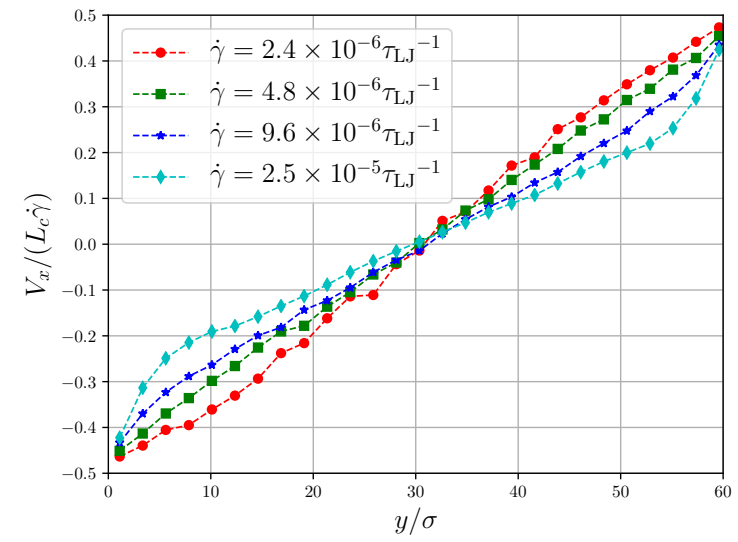

(a)

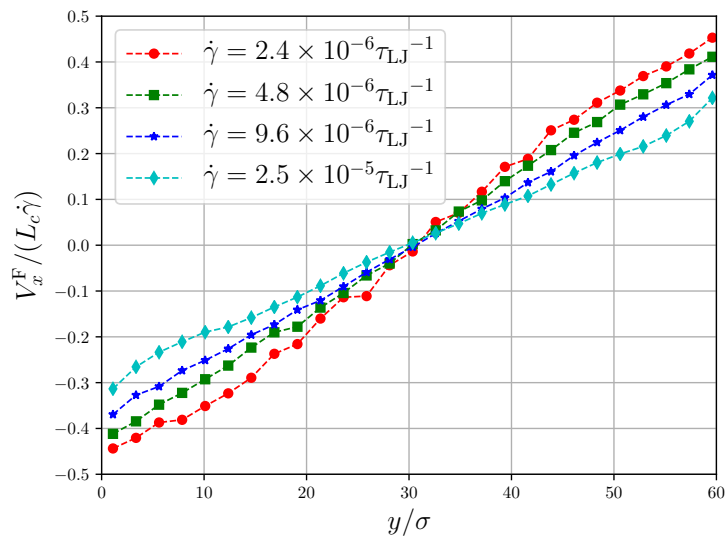

(b)

FIG. 6. Mean velocity profiles of (a) all monomers, $V_{x}$, and (b) only monomers forming free chains, $V_{x}^{\mathrm{F}}$. The profiles give the mean velocity in the flow direction normalized by the difference in the top and bottom plate velocities, $L_{c} \dot{\gamma}$, as a function of the monomer position within the channel, $y$. A selection of velocity profiles are included, corresponding to applied shear-rates which span the tethered chain coil-stretch transition. 


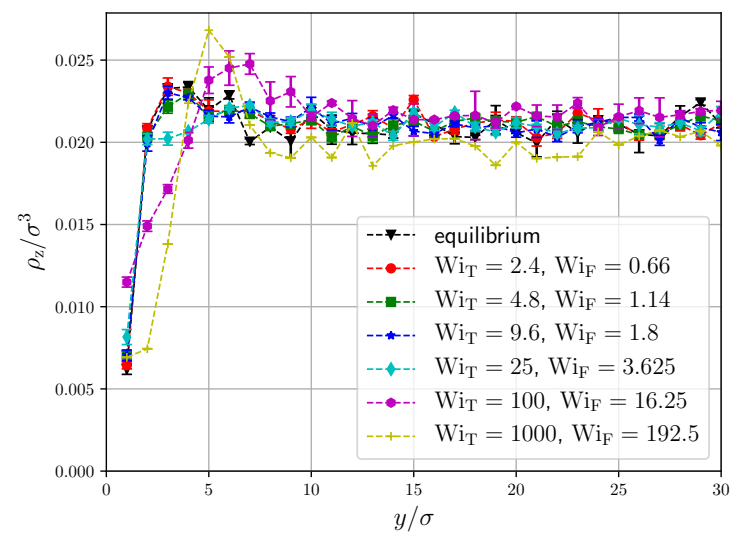

(a)

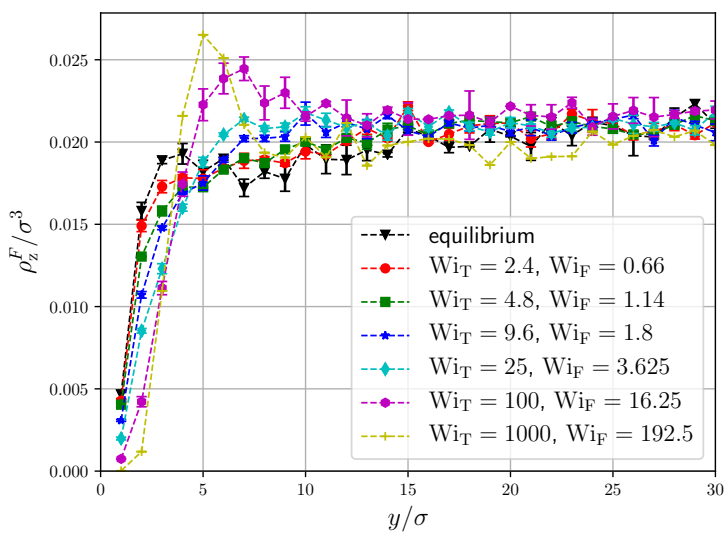

(b)

FIG. 7. (a) Channel density profile of all kinks, $\rho_{z}(y)$. (b) Channel density profile of all kinks on free primitive paths, $\rho_{z}^{\mathrm{F}}(y)$. Note that error bars are not available for gold crosses $\left(\mathrm{Wi}_{\mathrm{T}}=1000, \mathrm{Wi}_{\mathrm{F}}=192.5\right)$. [ $\mathrm{s}$ Throughout the $\mathrm{m} / \mathrm{s}$ and figures, should it be $\rho_{Z}$ with capital (and slanted) $Z$ for kink densities? i.e. $\rho_{Z}^{\mathrm{F}}$ and $\rho_{Z}(y)$ ?]

kinks at a distance greater than $25 \sigma$ from the surface contribute), $\rho_{Z}^{\text {center }}$, as a function of bulk shear-rate.

Figure $5 \mathrm{~b}$ shows that the density of kinks drops by only approximately $5-10 \%$ between $\dot{\gamma} \approx 1 \times$ $10^{-4} \tau_{\mathrm{LJ}}^{-1}$ and $\dot{\gamma} \approx 1 \times 10^{-3} \tau_{\mathrm{LJ}}^{-1}$ whilst the bulk viscosity has dropped by a factor of approximately $10^{-0.61} \approx$ 0.25 (75\% decrease). The kink data may be compared with an alternative atomistic model [54], which gives a drop in kink density of $\approx 20 \%$ at only $\mathrm{Wi}_{\mathrm{F}} \approx 10$. The notable drop of $\rho_{Z}^{\text {center }}$ in Fig. 5b occurs at a delay of an order of magnitude in $\mathrm{Wi}_{\mathrm{F}}$ with respect to the atomistic model, despite a qualitative agreement in the shear-rate dependence of viscosity as a function of $\mathrm{Wi}_{\mathrm{F}}$ between the models. From Fig. 4 we also see that in the channel center the free chain order parameter is $S_{x}^{\mathrm{F}} \approx 0.5$ at $\mathrm{Wi}_{\mathrm{F}}=16.25$ which matches very closely to the atomistic simulation at a similar Weissenberg number (see figure $7 \mathrm{~b}$ of [54]).

The lack of a direct correlation between the viscosity and the number of kinks per chain at low $\mathrm{Wi}_{\mathrm{F}}$ in the current simulation appears as a warning against generally correlating the number of kinks with viscosity away from equilibrium. It encourages further investigation into the general rheological significance of the number of kinks per chain away from equilibrium even for true bulk systems. We leave detailed considerations of the bulk case for future work, and in the following sections we instead concentrate on investigating more closely the qualitative changes in the characteristics of kinks involving free and tethered chains as a function of applied shear-rate. We can note at least that Fig. 14d shows that the kinks are only slightly more concentrated at the ends of free chains at $\mathrm{Wi}_{\mathrm{F}}=16.25$ compared to at equilibrium; suggesting perhaps that the flow induced alignment of free chains is more important for bulk shear-thinning than this measure of topological interactions. 


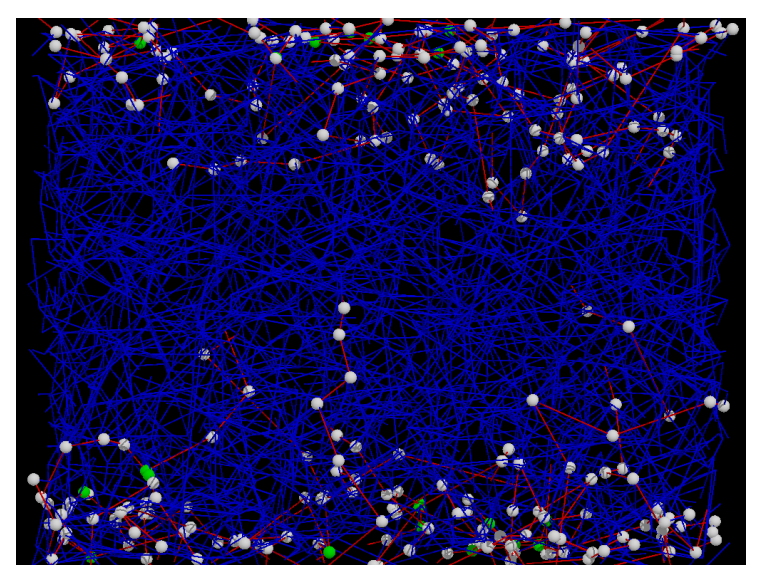

(a) Equilibrium

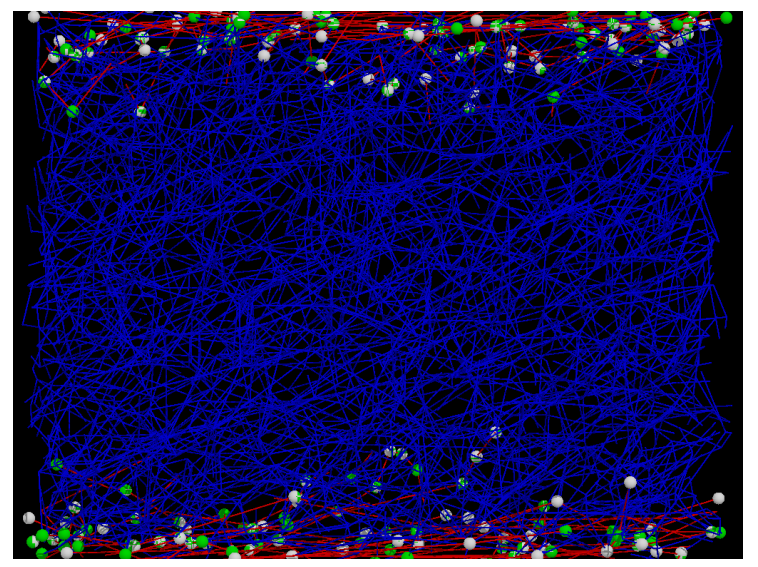

(c) $\mathrm{Wi}_{\mathrm{T}}=9.6, \mathrm{Wi}_{\mathrm{F}}=1.8$

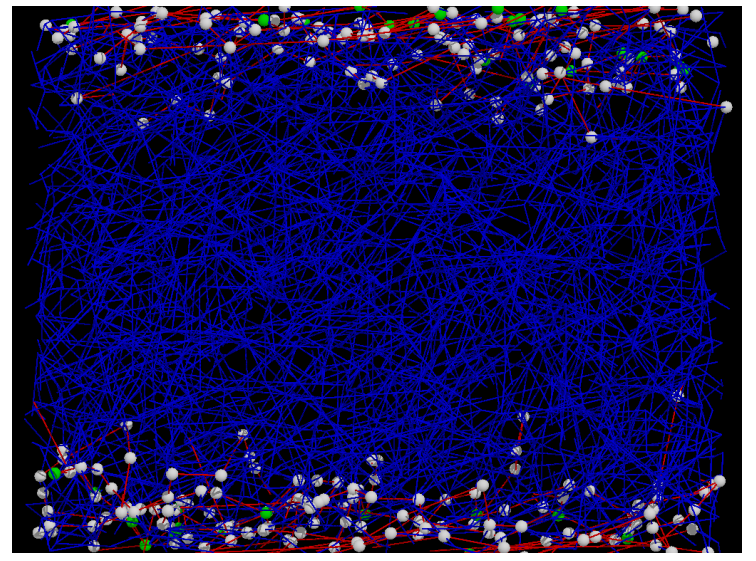

(b) $\mathrm{Wi}_{\mathrm{T}}=4.8, \mathrm{Wi}_{\mathrm{F}}=1.14$

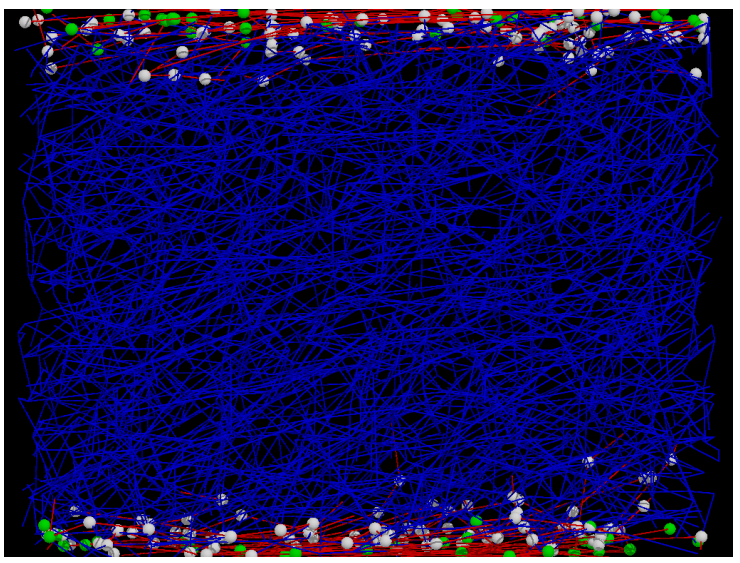

(d) $\mathrm{Wi}_{\mathrm{T}}=25, \mathrm{Wi}_{\mathrm{F}}=3.625$

FIG. 8. Snapshots of the complete set of primitive paths in the simulation box, using steady state shear simulations over a range of (a)-(d) four different flow-rates characterizing the tethered chain coil-stretch transition. Red lines: tethered chain primitive paths; blue lines: free chain primitive paths. Kinks in the primitive paths of tethered chains are indicated by spheres. White spheres: kinks due to the proximity of free chains (TF kinks); green spheres: kinks due to the proximity of tethered chains (TT kinks).

\section{B. Interface region behavior}

Figure 8 shows snapshots of all primitive path conformations in the simulation box, using a range of flow conditions characterizing the tethered chain coil-stretch transition. Tethered chain primitive paths are red whereas the primitive paths of free chains are blue. Figure 9 plots the root mean square of the tethered chain end-to-end vector projected onto the flow direction, at different applied shear-rates. At shear-rates greater than approximately $\dot{\gamma}=2.5 \times 10^{-5} \tau_{\mathrm{LJ}}^{-1}$ (Fig. 8) the tethered chains are so strongly deformed that it is possible for them to directly interact with their mirror images. As a result there may be some finite-size effects in the surface region behavior at such rates. The box dimension was chosen to avoid 


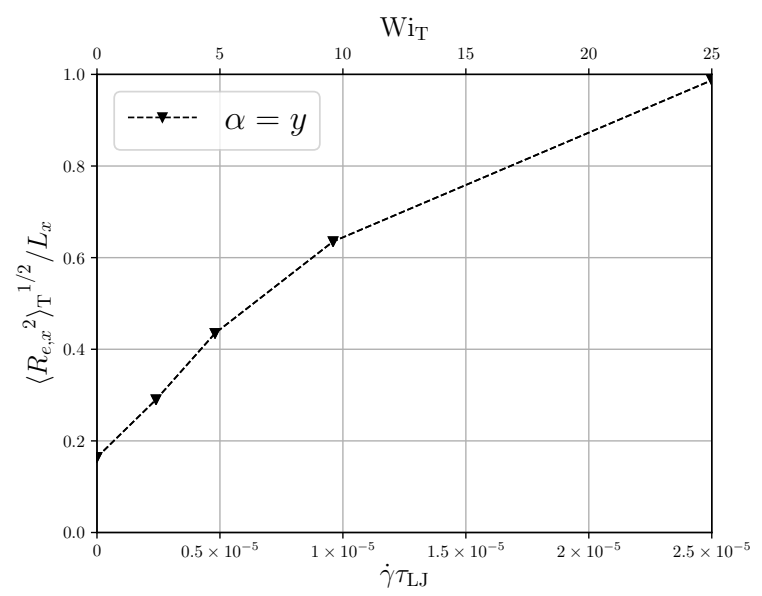

FIG. 9. Root mean square of the end-to-end vector projected onto the flow direction for tethered chains, $\left\langle R_{e, x}^{2}\right\rangle_{\mathrm{T}}{ }^{1 / 2} / L_{x}$. We normalize by the box length, $L_{x}$. Beyond an applied shear-rate of approximately $\dot{\gamma}=$ $2.5 \times 10^{-5} \tau_{\mathrm{LJ}}^{-1}$ the size of tethered chains can be greater than the box length, meaning that finite-size effects may occur.

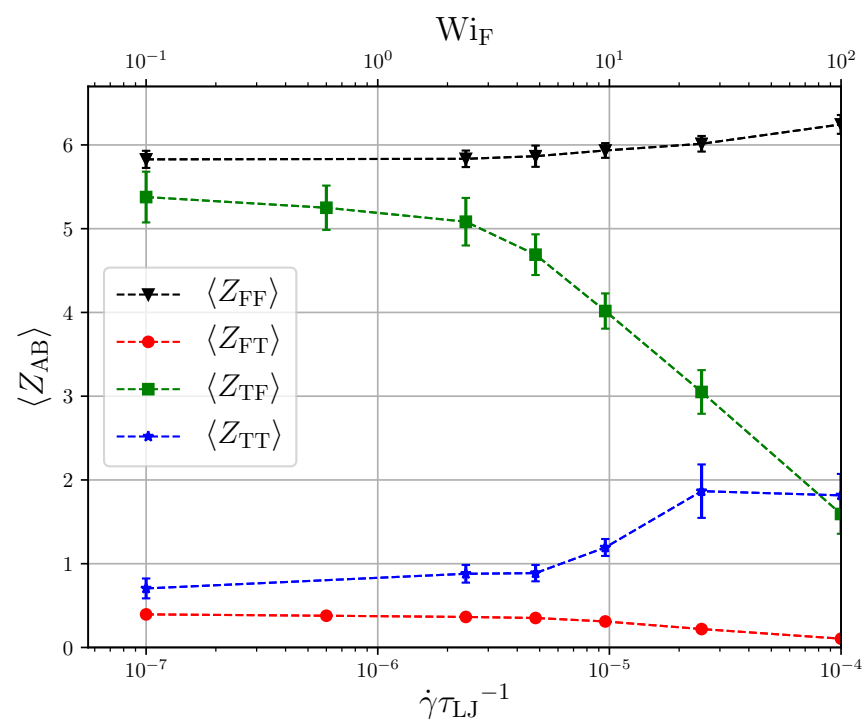

FIG. 10. Mean number of different types of kinks existing on a tethered or free chain primitive path as a function of applied shear-rate. $\left\langle Z_{\mathrm{AB}}\right\rangle$ stands for mean the number of kinks on $\mathrm{A}$ chains due to the proximity of a $\mathrm{B}$ chain, averaged over all A chains in the channel.

such issues at the most relevant range of shear-rates that characterize the coil-stretch transition. Figure 10 plots the shear-rate dependence of the mean numbers of kinks, (i) in a tethered chain primitive path due to the proximity of a free chain, $\left\langle Z_{\mathrm{TF}}\right\rangle$; (ii) in a free chain primitive path due to the proximity of a tethered chain, $\left\langle Z_{\mathrm{FT}}\right\rangle$; (iii) in tethered due to tethered, $\left\langle Z_{\mathrm{TT}}\right\rangle$; and (iv) in free due to free, $\left\langle Z_{\mathrm{FF}}\right\rangle$. At equilibrium, $\left\langle Z_{\mathrm{TF}}\right\rangle+\left\langle Z_{\mathrm{TT}}\right\rangle \approx\left\langle Z_{\mathrm{FT}}\right\rangle+\left\langle Z_{\mathrm{FF}}\right\rangle:$ the mean number of kinks in tethered chains is almost identical to that 


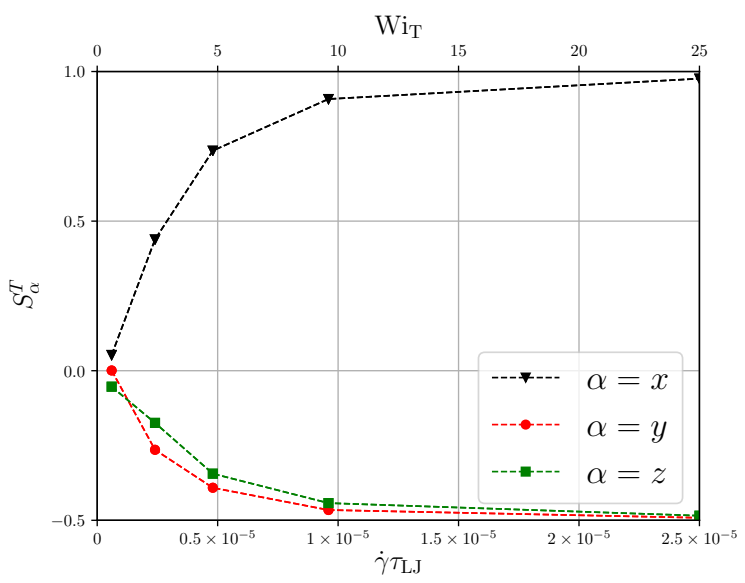

(a)

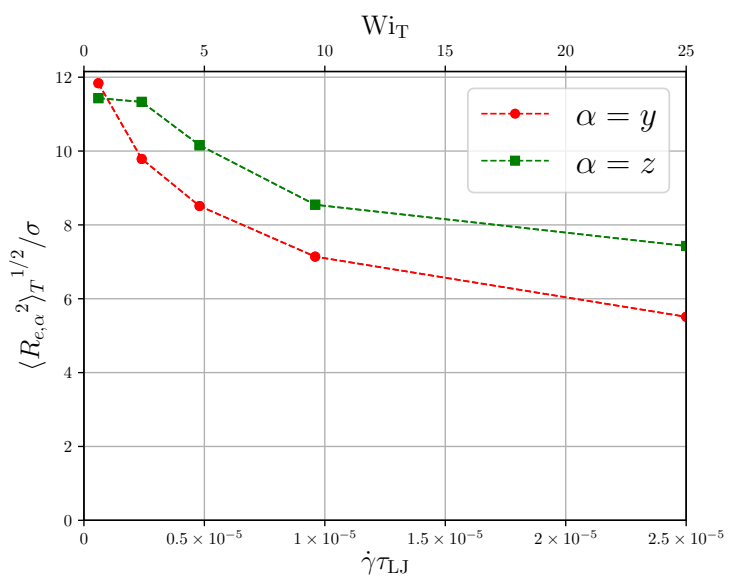

(b)

FIG. 11. (a) Directional orientation order parameters of the end-to-end unit vector of tethered chains, $S_{\alpha}^{\mathrm{T}}$, versus applied shear-rate; (b) Root mean square of components of the end-to-end vectors of tethered chains normal to the velocity field, i.e., in flow gradient $\hat{\mathbf{y}}$ and vorticity $\hat{\mathbf{z}}$ directions, versus applied shear-rate.

of free chains.

In Fig. $11 \mathrm{a}$ three orientation order parameters $\left(S_{\alpha}^{\mathrm{T}}, \alpha=x, y, z\right)$ for the corresponding components of the end-to-end unit vector of the tethered chains are plotted as a function of applied shear-rate. We defined $S_{\alpha}^{\mathrm{T}}$ already in Eq. 11). The chain alignment in the flow direction is already characterized by orientation parameter $S_{x}^{\mathrm{T}} \approx 0.9$ at $\mathrm{Wi}_{\mathrm{F}}=1.8\left(\mathrm{Wi}_{\mathrm{T}}=9.6\right)$, so that the tethered chain flow alignment occurs before any strong deformation of free chains. Fig. 10 shows that $\left\langle Z_{\mathrm{TF}}\right\rangle$ and $\left\langle Z_{\mathrm{FT}}\right\rangle$ begin to decrease as soon as the tethered chain begins to orient. $\left\langle Z_{\mathrm{TT}}\right\rangle$ initially begins to increase and then stagnates beyond the coilstretch transition. Figure $12 \mathrm{a}$ plots the corresponding bead density profiles for the tethered chain species, which may be compared with recent experimental results for the same observable [10]. The transition from isotropy to flow alignment (coil-stretch transition) takes place over a much more narrow range of shear-rates for the tethered chains compared to the free chains (Fig. 4), but it does not coincide with any sudden large drop in the number of kinks involving free and tethered chains which would be expected for complete interfacial disentanglement as assumed in the earliest scaling model [19]. For the interfacial disentanglement, the early scaling models emphasized the importance of the surface normal component of the tethered chain dimension rather than the angle of the end-to-end vector with respect to the surface. Figure $11 \mathrm{~b}$ plots the root mean square of the end-to-end vector projected onto directions normal to the flow. For the largest shear-rates considered, the root mean square of the end-to-end vector projected in the surface normal direction is similar to the root mean square of the end-to-end vector of a bulk segment with entanglement bond number, $N_{e}$, at equilibrium (taking for example $8 \sigma$ as a modest estimate for the root 


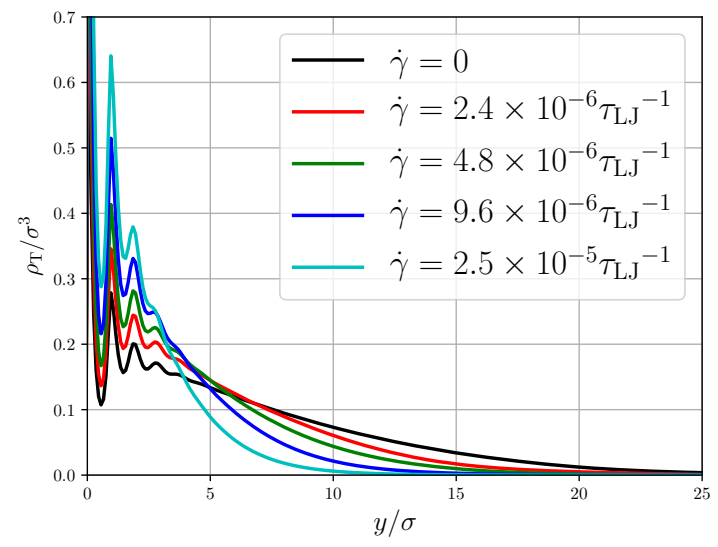

(a)

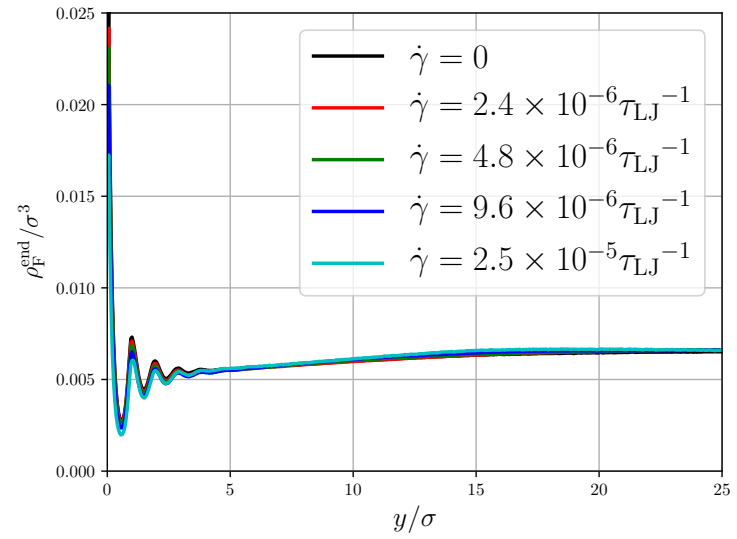

(b)

FIG. 12. (a) Tethered bead number density, $\rho_{\mathrm{T}}(y)$; (b) Number density of end beads of free chains, $\rho_{\mathrm{F}}^{\text {end }}(y)$. Both observables are given as a function of the distance to the surface, $y$, at different applied shear-rates spanning the tethered chain coil-stretch transition.

mean square of the entanglement segment end-to-end vector corresponding to $N_{e} \approx 40$ [16, 58]). Therefore we are clearly within the scaling regime for surface disentanglement of Brochard and De Gennes. The gradual drop in TF kinks (surface 'disentanglement') apparent in Fig. 10 is consistent with the assumptions of the more detailed scaling models [20, 21, 26].

Figure 13 plots the root mean square of the components of end-to-end vectors of tethered chain subsegments comprising 32 bonds, as a function of the index $\ell$ of the first monomer in the segment. The first segment begins at the grafted monomer $(\ell=0)$ and the final segment begins at $\ell=224$. From Fig. 13a we see that the orientations of the tethered chains have a trumpet like quality as predicted by Ajdari et al. [20]. Following this observation it is natural to ask whether this anisotropic degree of deformation corresponds with a non-uniform occupational density of kinks along the tethered chain primitive path contour. To address this question first we must define a contour parameter for the primitive path.

\section{Kink densities as a function of the primitive path contour variable}

At the beginning of the $\mathrm{Z} 1$ algorithm [11-13], kinks are located at the positions of the interior chain beads. Together with the two 'ghost nodes' occupying the end bead positions, which remain fixed in space and conserved throughout the algorithm, the kinks are more generally 'nodes'. At the beginning of the algorithm there are then 257 nodes and 255 kinks in each primitive path in the current work.

Each node is initially assigned a contour label, given by the index of the bead that coincides with the 


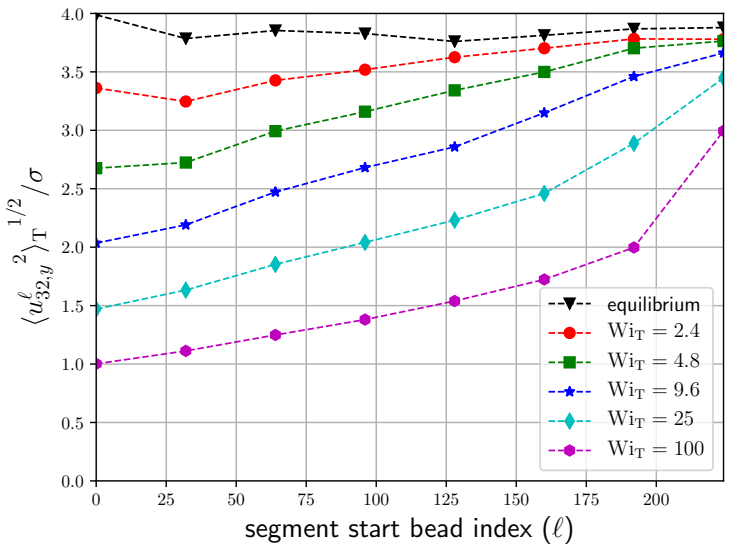

(a)

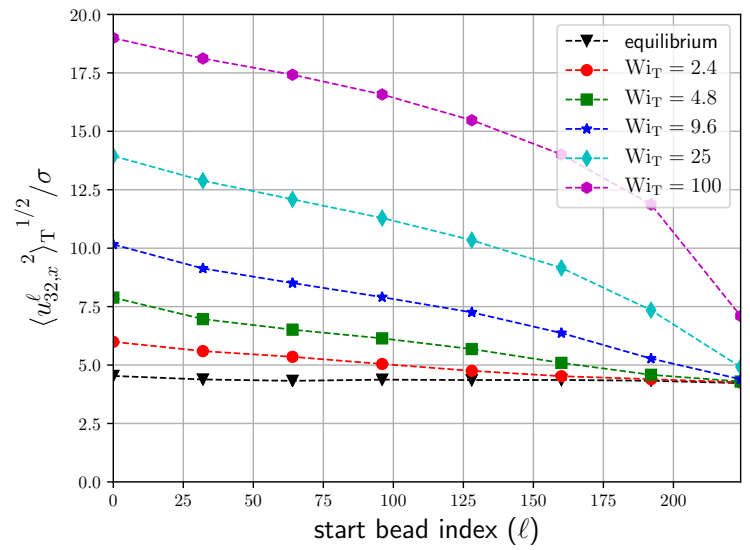

(b)

FIG. 13. Root mean square of components of the end-to-end vector of sub-segments comprising $\Delta \ell=32$ bonds in the tethered chain, as a function of the index $\ell$ of the first monomer in the sub-segment. The grafted sub-segment starts at monomer $\ell=0$ and the final sub-segment starts at monomer $\ell=224$. Data shown for several applied shear-rates.

node. As the algorithm progresses many nodes disappear as the chain contour length is reduced. When nodes are displaced in space they keep the contour label that they were initially assigned. When a primitive path is shortened, new nodes may be created on the chain contour (see Ref. [12]). If a new node is created such that it becomes the $j$ th node along the chain contour, then it is assigned a contour label, $\ell_{j}$, intermediate between $\ell_{j-1}$ and $\ell_{j+1}$ :

$$
\ell_{j}=\ell_{j-1}+\left(\ell_{j+1}-\ell_{j-1}\right) \frac{\left|s_{j}-s_{j-1}\right|}{\left|s_{j+1}-s_{j}\right|+\left|s_{j}-s_{j-1}\right|}
$$

where $s_{j}$ is the $j$ th node position in space. Note that this means that $\ell_{j}$ is not necessarily an integer. When the $\mathrm{Z} 1$ algorithm is finished the final set of kink contour labels, $\left\{\ell^{Z}\right\} \backslash\left\{\ell_{0}, \ell_{Z+1}\right\}$, [\& I replaced $Z-1$ by $Z+1$ here and below] in each chain is recorded. Here $Z$ is the final number of kinks in a primitive path. Note that the ghost node contour labels, $\left\{\ell_{0}=0, \ell_{Z+1}=n\right\}$, are excluded from the set of kinks. We can examine the progressive drop in the mean number density of kinks along the primitive paths using the sets of contour labels. A contour label is bounded by the start and end values the contour variable may take: $0 \leq \ell \leq n$. We are interested in exploring the possibility of correlations between the density of kinks at different values of the contour variable, the real chain orientations, and the corresponding flow behavior. We note that alternative parameterizations of the primitive path contour are possible, and it would be desirable to examine whether such alternatives give similar qualitative results for kink occupational densities. The most easily accessible alternate definition is the arc length of the primitive path considered as a polygon in space, where the information about original beads is completely ignored. 
In Fig. 14 we plot kink occupational densities in units of the inverse contour distance, as a function of the contour variable, $\ell$. Entanglements are frequently interpreted as binary interactions between different chains [53]. Kinks in the network of primitive paths do not always fit this picture: it is possible that a kink occurs in a primitive path due to the proximity of a straight part of another primitive path, although it is also possible that a kink on one path can be associated with a corresponding kink on another path. In the Z1 algorithm, a kink is always associated with a unique contour variable label for the part of a secondary primitive path whose proximity led to the kink, whether or not this contour label corresponds to the position of a kink. We define $\left\langle z_{\mathrm{FT}}^{\text {tethered }}(\ell)\right\rangle$ as an occupational density of instances in which the proximity of a tethered path at contour variable around $\ell$, to a free chain path, resulted in a kink on the free chain path. The integral of this quantity satisfies the relation: $48 / 628 \int d \ell\left\langle z_{\mathrm{FT}}^{\text {tethered }}(\ell)\right\rangle=\int d \ell\left\langle z_{\mathrm{FT}}(\ell)\right\rangle=\left\langle Z_{\mathrm{FT}}\right\rangle$. Here $\left\langle z_{\mathrm{FT}}(\ell)\right\rangle$ is an occupational density of kinks around contour position $\ell$ in a free chain primitive path due to the proximity of part of a tethered chain primitive path. We also define $\left\langle z_{\mathrm{TF}}(\ell)\right\rangle$ as an occupational density of kinks around contour position $\ell$ in a tethered chain primitive path due to the proximity of part of a free chain primitive path. This density satisfies $\int d \ell\left\langle z_{\mathrm{TF}}(\ell)\right\rangle=\left\langle Z_{\mathrm{TF}}\right\rangle$. We plot $\left\langle z_{\mathrm{TF}}(\ell)\right\rangle$ and $\left\langle z_{\mathrm{FT}}^{\text {tethered }}(\ell)\right\rangle$ in Fig. 14a, which shows that they both have very similar density profiles at all shear-rates, in agreement with a binary picture of entanglements. Note however that in general $\left\langle z_{\mathrm{FT}}^{\text {tethered }}(\ell)\right\rangle$ is larger than $\left\langle z_{\mathrm{TF}}(\ell)\right\rangle$ near to the free ends of primitive paths.

At equilibrium, Fig. 14a shows that the density of TF kinks is close to uniform along the contour, with only a small drop near the tethered end (the contour variable corresponding to the tethered end is $\ell=0$ ). Kinks are no longer distributed equally along the contour of tethered chains under (strong enough) shear. The contour positions from which kinks due to free chains are lost from tethered paths under shear, with respect to at equilibrium, correlate with the contour positions of the tethered chain sub-segments which have a decreased size normal to the surface (see Fig. 13a). However it does not appear clear from this data whether the drop in kinks is only a function of chain segment orientation or whether it also depends on the position of the segment relative to the surface. I.e. would the situation change for a tethered chain pulled through a melt far from the surface. Figure 15 plots the mean surface normal position of the $\ell$ th bead of tethered chains.

Figure $14 \mathrm{~b}$ plots the contour occupational densities of kinks along the primitive path of free chains that are due to the proximity of a tethered chain, $\left\langle z_{\mathrm{FT}}(\ell)\right\rangle$, at different applied shear-rates. We find that as the tethered chain aligns with the surface there is an increasing relative probability of forming a kink near the end of a nearby free chain with respect to forming one near the center of the free chain. The end bead density profile of free chains, plotted in Fig. $12 \mathrm{~b}$, is found to be approximately independent of shear-rate: the distribution of chain ends is largely unchanged with respect to at equilibrium for all shear-rates of interest. 


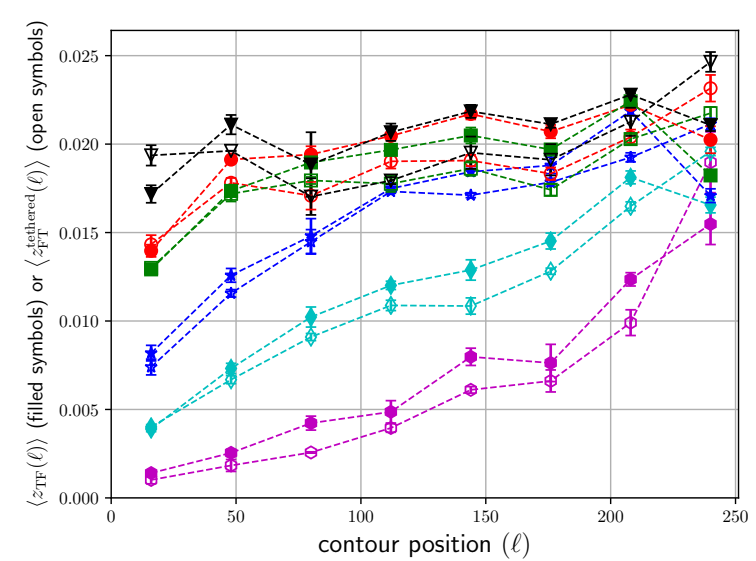

(a)

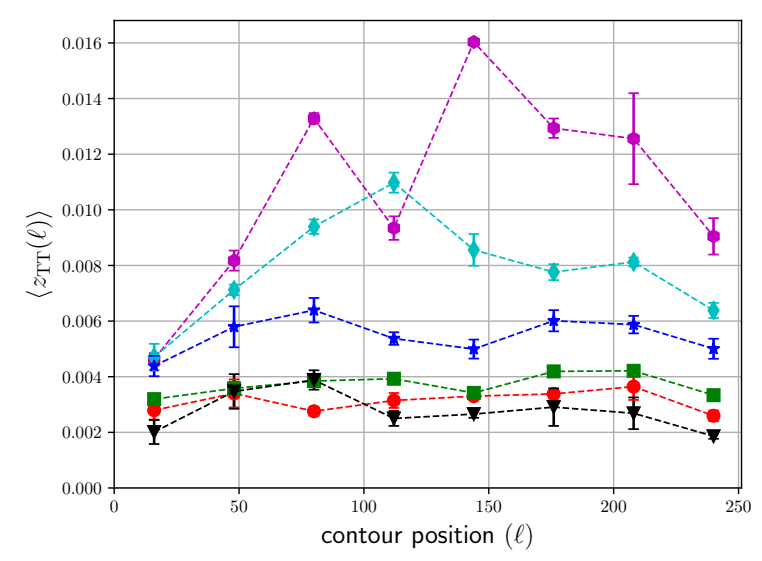

(c) [? strange that one error bar is so large]

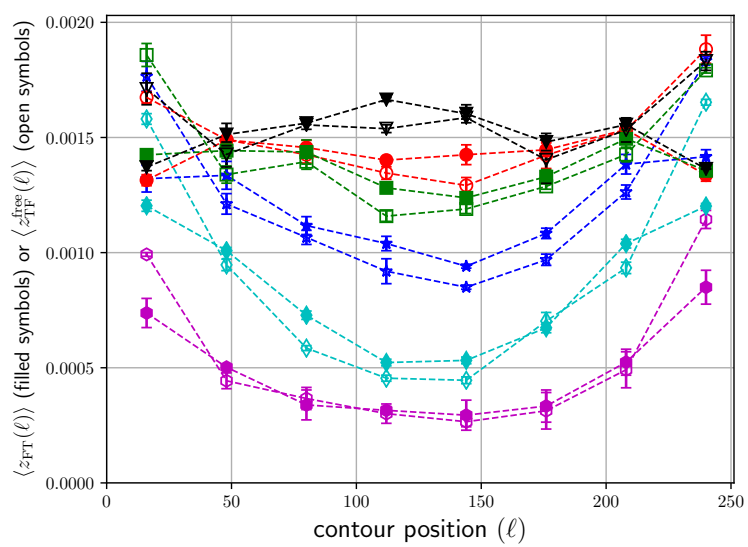

(b)

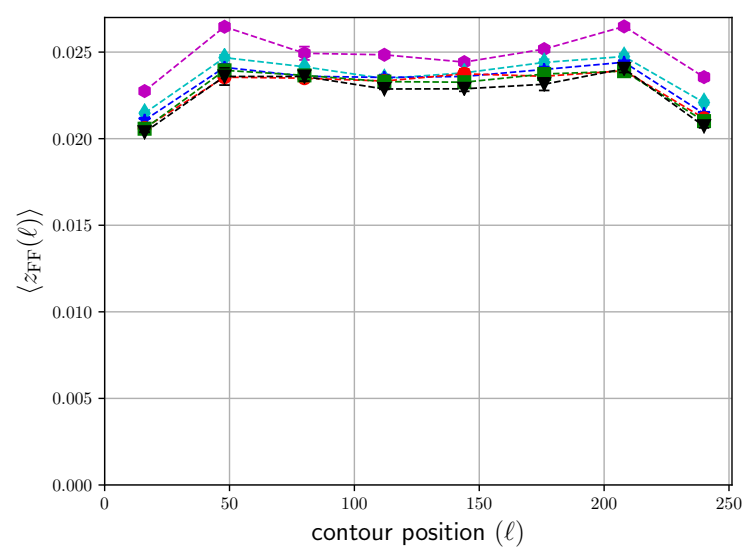

(d)

FIG. 14. Mean local occupational densities of kinks along primitive path contours, averaged over primitive path segments with size $\Delta \ell=32$ (to avoid a certain noise level). Different plots correspond to different applied shearrates. Black triangles: $\dot{\gamma}=0$; red circles: $\dot{\gamma}=2.4 \times 10^{-6} \tau_{\mathrm{LJ}}^{-1}$; green squares: $\dot{\gamma}=4.8 \times 10^{-6} \tau_{\mathrm{LJ}}^{-1}$; blue stars: $\dot{\gamma}=9.6 \times 10^{-6} \tau_{\mathrm{LJ}}^{-1}$; cyan diamonds: $\dot{\gamma}=2.5 \times 10^{-5} \tau_{\mathrm{LJ}}^{-1}$; magenta hexagons: $\dot{\gamma}=1 \times 10^{-4} \tau_{\mathrm{LJ}}^{-1} .(\mathbf{a})\left\langle z_{\mathrm{TF}}(\ell)\right\rangle$ (filled symbols) is the mean local density of kinks (with contour variable around $\ell$ ) in tethered chain primitive paths due to the proximity of a free chain. $\left\langle z_{\mathrm{FT}}^{\text {tethered }}(\ell)\right\rangle$ (open symbols) is the mean local density of instances in which the proximity of a tethered chain path (at contour variable around $\ell$ ) results in a kink on a free chain path. $(\mathbf{b})\left\langle z_{\mathrm{FT}}(\ell)\right\rangle$ (filled symbols) is the mean local density of kinks on free chain primitive paths due to the proximity of a tethered chain. $\left\langle z_{\mathrm{TF}}^{\text {free }}(\ell)\right\rangle$ (open symbols) is the mean local density of instances in which the proximity of a free chain path results in a kink on a tethered chain path. (c) $\left\langle z_{\mathrm{FF}}(\ell)\right\rangle$ (filled symbols) is the mean local density of kinks in free chain primitive paths due to the proximity of a free chain. $(\mathbf{d})\left\langle z_{\mathrm{TT}}(\ell)\right\rangle$ (filled symbols) is the mean local density of kinks in tethered chain primitive paths due to the proximity of a tethered chain. 


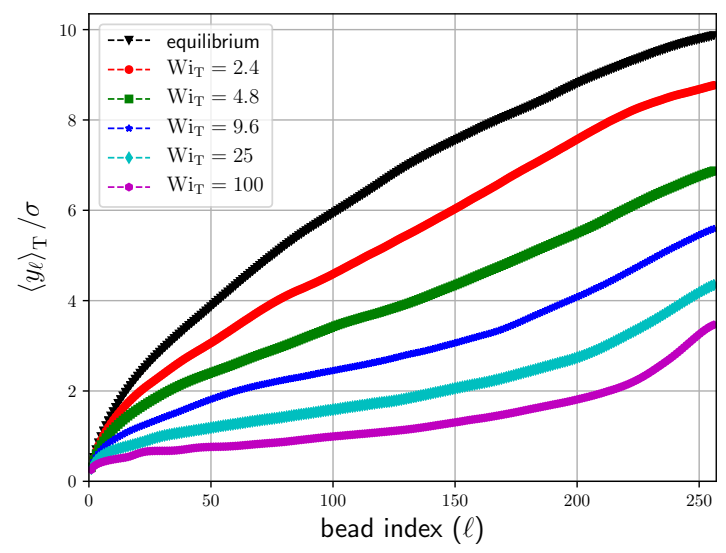

FIG. 15. Mean surface normal position, $\left\langle y_{\ell}\right\rangle_{\mathrm{T}} / \sigma$, of the $\ell$ th bead of tethered chains at different applied shear-rates.

An excess of end beads within a monomer size from the surface, followed by a corresponding depletion until a distance of approximately the molecular size from the surface, has been theoretically predicted by Matsen and Mahmoudi [59], using a system completely made up of free chains. We find that this characterizes the end density plot well (Fig. 12b), although it is difficult to immediately discern the excess at the surface from the plots due to the oscillations in bead density. It can be immediately verified that the mean end bead density of free chains within a molecular distance from the surface, $\left\langle\boldsymbol{R}_{e, \text { bulk }}^{2}\right\rangle^{1 / 2} \approx 17.5 \sigma$, matches the channel center value: the excess and depletion cancel one another. It is expected that an excess in the end segment density of free chains at the surface will lead to an enhanced constraint release rate of free chains acting on tethered chains as the tethered chain becomes aligned against the surface.

Figure $14 \mathrm{~d}$ plots the contour occupational density of kinks on free paths due to other free paths averaged over the whole channel, $\left\langle z_{\mathrm{FF}}(\ell)\right\rangle$. The integral of this quantity over the complete contour satisfies $\int d \ell\left\langle z_{\mathrm{FF}}(\ell)\right\rangle=\left\langle Z_{\mathrm{FF}}\right\rangle$. Unlike FT kinks (Fig. 14b), there is little difference in the contour density profiles for FF kinks over the range of shear-rates characterizing the tethered coil-stretch transition. Figure $14 \mathrm{c}$ plots the contour occupational density of kinks on tethered paths due to other tethered paths, $\left\langle z_{\mathrm{TT}}(\ell)\right\rangle$. At equilibrium $\left\langle z_{\mathrm{TT}}(\ell)\right\rangle$ is roughly uniform across the path contour. At shear-rates beyond the coil-stretch transition the profile becomes increasingly non uniform.

A uniform density of entanglements along the chain contour is frequently assumed in theoretical models [14, 60] even away from equilibrium [30, 32]. Our results in this section suggest that this assumption is incorrect for the entangled interfacial chains if we directly associate primitive path kinks with entanglements. Figure $14 \mathrm{~d}$ indicates that this assumption is more appropriate for free-free (FF) kinks, although preliminary results at faster rates indicates some decrease in kink density in the contour center for free chains. This 
may be the first time that the density of primitive path kinks as a function of contour position has been investigated for a system of linear KG chains, although a contour density plot of an alternative measure of entanglements has been given by Likhtman and Ponmurugan at equilibrium [61], which is qualitatively similar to Fig. 14d. Recently the kink density along the primitive paths of lamellae forming block copolymers has also been investigated [62].

\section{Slip length and surface friction}

A common measure of slip is the 'slip length'. The slip length may be interpreted as the distance beyond the surface at which an extrapolation of the fluid velocity profile reaches the wall velocity. The velocity profile is extrapolated from a point in the fluid at a distance $a$ from the surface, moving with velocity $V_{s}$ with respect to the surface:

$$
L_{\text {slip }}=\frac{V_{s}}{\dot{\gamma}_{\text {bulk }}}-a \approx \frac{V_{s}}{\dot{\gamma}_{\text {bulk }}} ; \quad a / L_{\text {slip }} \approx 0
$$

It is often assumed that $a / L_{\text {slip }} \approx 0$ which simplifies the above definition [19, 24]. Alternatively, the 'slip length' may be strictly defined as $L_{\text {slip }}=V_{s} / \dot{\gamma}_{\text {bulk }}$ for all cases, whereupon its interpretation becomes a priori unclear. We call $V_{s}$ the slip velocity; there are several possibilities for the definitions of $\dot{\gamma}_{\text {bulk }}$ and also $V_{s}$ which we will discuss in the following. [ $\mathrm{s}$ I here assumed that the next paragraph will become part of the $\mathrm{m} / \mathrm{s}$. The present paragraph is not $100 \%$ clear. How is $L_{\text {slip }}$ defined in this work? By the ratio without the $a$ ? Then the word 'unclear' is not well chosen, to my opinion, as we are going to shed some light onto it. Using a finite $a$ which must be, as part of the definition, small compared to $L_{\text {slip }}$ ? In that case this assumption does not simplify the definition, but is part of it .. Is $L_{\text {slip }}$, as the def. stands, a function of $a$ ? If not, can it be written as $\lim _{a \rightarrow 0}\left(V_{s} / \dot{\gamma}_{\text {bulk }}\right)-a=$ ?]

A degree of arbitrariness arises in the choice of the shear-rate used to extrapolate the slip velocity to zero $\left(\dot{\gamma}_{\text {bulk }}\right.$ in Eq. (3)). In a simulation study of low molecular weight chains Priezjev and Troian [63] define the slip length by extrapolating the fluid velocity to zero using the final three data points of the velocity profile nearest the surface. In this portion of the fluid the shear-rate deviates appreciably from the channel center. Experimentally the velocity field may also be resolved close to the surface [23, 24, 64]. Léger et al. [24] use a channel of diameter $d$, within which a velocity field is generated via a no-slip top plate moving at velocity $V_{t}$. The fluid slips past the stationary bottom plate with slip velocity $V_{s}$. In this case it is assumed than the velocity profile in the channel remains linear so that the slip length is found from the relation: 
$L_{\text {slip }}=V_{s} d /\left(V_{t}-V_{s}\right)$ (for very wide channels of course this definition will tend to our center channel definition). Our procedure differs with respect to both these cases in that $\dot{\gamma}_{\text {bulk }}$ is defined in the channel center. ]

At low Reynolds numbers, the simple planar Couette geometry enforces a balance of shear stresses in the channel: $V_{s} \xi_{s}=\dot{\gamma}_{\text {bulk }} \eta_{\text {bulk }}$, where $\xi_{s}$ is a surface friction coefficient. We may define the surface friction coefficient via:

$$
\xi_{s}=\eta_{\text {bulk }} / L_{\text {slip }}
$$

provided that the slip length strictly satisfies: $L_{\text {slip }}=V_{s} / \dot{\gamma}_{\text {bulk }}$. [o Sounds strange for a typical reader, as we just wrote that there is something unclear in that case. Can 'strictly satisfied' be replaced by 'satisfied'?] The bulk viscosity, $\eta_{\text {bulk }}$, is given in Fig. 5a. At this point we can consider how the interpretation of $\xi_{s}$ depends on our definition of $L_{\text {slip }}$ (or more precisely $V_{s}$, since we keep $\dot{\gamma}_{\text {bulk }}$, and the relation Eq. (4) fixed). If we are interested in a friction force, $V_{s} \xi_{s}$, acting between the permanently grafted surface chains and the free chains, one choice is to define the slip velocity from the free chain monomeric velocity profile, Fig. 6b. using the data point closest to the surface. We denote this free monomer slip velocity by $V_{s}^{\mathrm{F}}$, appropriate for describing the surface friction using completely adsorbing surfaces. The data point closest to the surface in Fig. 6averages the velocities of monomers that are located within a distance of approximately $2 \sigma$ from the surface. The slip length found using Eq. 3 with $V_{s}=V_{s}^{\mathrm{F}}$ is plotted in Fig. $16 \mathrm{~b}$ (red circles).

For other systems with only partially adsorbing surfaces (different from the current case), it is clear that chain desorption may influence the surface friction far from equilibrium. In such a case it would seem more natural that the slip velocity is defined using the velocity profile of all monomers, Fig. 6b. We denote such a total monomer slip velocity by $V_{s}^{\text {all }}$. The slip length defined using Eq. 3 with $V_{s}=V_{s}^{\text {all }}$ is plotted in Fig. 16b (green squares). Experimentally this is naturally how the slip velocity is defined [23, 24, 64]. For shear-rates spanning the coil-stretch transition $\left(\mathrm{Wi}_{\mathrm{T}}<25\right)$, the slip length found using $V_{s}^{\text {all }}$ increases at a weaker rate compared to the slip length found using $V_{s}^{\mathrm{F}}$. This is unsurprising since the monomers in the region within $2 \sigma$ from the surface are progressively more likely to be part of a tethered chain, travelling at the surface velocity. For the current case of a simulation using reflective surfaces and permanently grafted chains this choice for the slip velocity seems inappropriate.

So far we have considered two definitions of the slip velocity; the first may be judged more appropriate for non-adsorbing surfaces with permanently grafted chains, and the second more appropriate for partially adsorbing surfaces.

A possible criticism of both choices is that they depend only on information of the fluid locally at the surface, even though it could be argued that the surface friction force acts via the adsorbed chains which 
are extended objects in space. Considering the permanently grafted case, tethered chains are extended from the surface but move with the surface velocity. We could then interpret the grafted chains as part of the 'wall', so that the 'wall' extends beyond the surface. How then is it appropriate to define the slip velocity via the fluid velocity immediately at the surface when the 'wall' is an object which extends beyond this point? Certainly we may observe from Fig. 6 that the shear-rate deviates from the center channel value much further from the surface than $2 \sigma$; At the very lowest shear-rate in Fig. 6, deviations from the linear velocity profile at the channel center extend until approximately a molecular size from the surface. [ $\$$ How deviations from the linear profile can be associated with a surface 'friction' or changes in fluid properties near the surface is not clear. One may simply argue that the surface (tethered chains) and the fluid are mixed together, and that resultantly none of the definitions of the surface friction we use can clearly related to a surface friction force. Going beyond this statement is I think well beyond the scope of the work. ]

This consideration suggests an alternative definition of the slip length. Since the channel in our simulation is symmetric, the slip velocity may be defined by:

$$
2 V_{s}=L_{c} \dot{\gamma}-\left(L_{c}-2 a\right) \dot{\gamma}_{\text {bulk }}
$$

In Eq. (5) the slip-velocity is given by the velocity of the fluid at the point relative to the surface at which the shear-rate first deviates from the center channel value, a distance $a$ from the surface. In this case the slip length, Eq. (3), becomes:

$$
L_{\text {slip }}=\frac{L_{c} \dot{\gamma}-\left(L_{c}-2 a\right) \dot{\gamma}_{\text {bulk }}}{2 \dot{\gamma}_{\text {bulk }}}-a=\frac{L_{c}}{2}\left(\frac{\dot{\gamma}}{\dot{\gamma}_{\text {bulk }}}-1\right)
$$

The black triangles in Fig. $16 \mathrm{~b}$ correspond to this definition of the slip length. It gives very similar values to the definition which uses $V_{s}^{\mathrm{F}}$ during the coil stretch transition. However at the lowest shear-rates it drops more rapidly and becomes negative, meaning that the extrapoloated velocity reaches zero inside the fluid. In Fig. 16a the ratio $\dot{\gamma}_{\text {bulk }} / \dot{\gamma}$ that is used in Eq. (6) is plotted as a function of applied shear-rate. For all three definitions of the slip length, as the tethered chain orients the slip length increases before the bulk shear-thinning regime is entered whereupon the slip length decreases.

Although in the case of Eq. (6) the slip length is independent of $a$, the relationship between the slip velocity as defined in Eq. (5) to the slip length is only approximately given by $L_{\text {slip }}=V_{s} / \dot{\gamma}_{\text {bulk }}$ for the case that $a / L_{\text {slip }} \approx 0$. More generally if we define the slip length using Eq. $(6)$, then define the slip velocity via $V_{s}^{\text {extrap }}=L_{\text {slip }} \dot{\gamma}_{\text {bulk }}$, this slip-velocity corresponds to the value of the bulk velocity field extrapolated to the surface (which is different to slip velocity in Eq. (5)). Therefore the surface friction, $\xi_{s}$, found from the relations Eq. 4. and 6 corresponds to the surface friction force $\xi_{s} V_{s}^{\text {extrap }}$.

We conclude finally that the idea that any of the three friction coefficients considered here can be interpreted unambiguously as a surface friction is highly questionable. Bearing this warning in mind we choose 
to comment only on the qualitative correlations between the friction coefficients and the state of the fluid under different flow conditions.

The three definitions of the surface friction, Eq. (4), corresponding to the three definitions of slip length (Fig. 16b are plotted in Fig. 16c as a function of applied shear-rate. The most rapid drop in surface friction universally occurs before $\dot{\gamma}=9.6 \times 10^{-6} \tau_{\mathrm{LJ}}$, during the coil-stretch transition, at the beginning of the drop in tethered-free kinks (Fig. 10). Figure 14 shows that, although only a relatively small proportion of the tethered-free kinks are lost by $\dot{\gamma}=9.6 \times 10^{-6} \tau_{\mathrm{LJ}}$, those which are lost were almost exclusively located in the half of the tethered contour closest to the tethered end, or the center chain for the case of free chains. The

friction defined using the velocity at the surface extrapolated from the channel center, $V_{s}^{\text {extrap }}$, fails strongly in its interpretation as a surface friction at the lowest shear-rate $\left(\dot{\gamma}=2.4 \times 10^{-6} \tau_{\mathrm{LJ}}\right)$. At this shear-rate the friction coefficient is negative since the extrapolated velocity at the surface is greater than the wall velocity. Finally Fig. $16 \mathrm{~d}$ shows that the combined effect of bulk shear thinning and surface slip is too weak to lead to a non-monotonic flow curve such that at this chain length stable dynamical behavior is expected across the film. In Fig. 16d the black circles correspond to the experimentally observable flow curve, whereas green triangles corresponds to the flow curve in terms of the bulk shear-rate.

\section{CONCLUSION}

We have performed non-equilibrium shear flow simulations which have both tested existing ideas regarding surface disentanglement and revealed new phenomena.

Firstly, if the number of kinks in primitive paths is in some way correlated with the number of 'entanglements' (even away from equilibrium), the simulations validate the idea of a surface disentanglement resulting from the coil-stretch transition of grafted chains. However, we find that in the non-linear flow regime the density of kinks does not necessarily correlate with dynamic viscosity in the bulk. It would be interesting to investigate the behavior of dynamic definitions of entanglements [16, 61] away from equilibrium, and their relation to primitive path kinks, even for true bulk chains. At equilibrium, the survival probability of 'long lived close contacts' has been demonstrated to be proportional to the linear stress relaxation function [18]. However such dynamic measures of entanglements are computationally expensive to investigate for non-equilibrium cases.

In addition to a quantitative drop in tethered-free kinks associated with the coil-stretch transition of tethered chains, Fig. 14a suggests the possibility of a drop in the rheological significance of tethered-free kinks, due to the increased relative probability that kinks in tethered primitive paths that are due to the close proximity of free paths have a contour position near the free end following surface alignment. This 


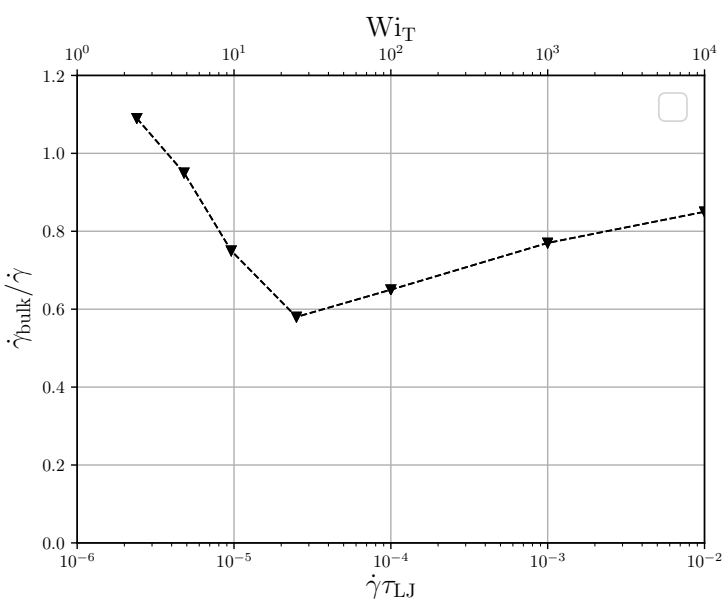

(a) Ratio of shear-rates (bulk to applied).

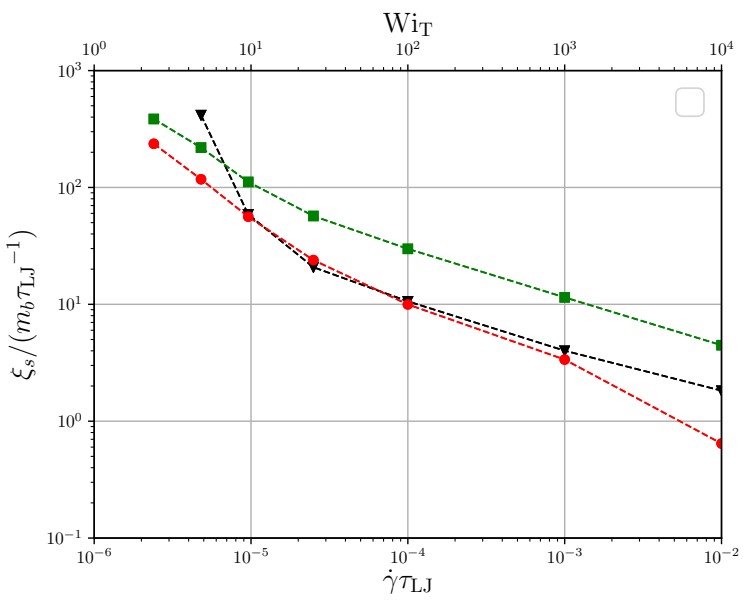

(c) Surface friction, $\xi_{s}$ (three definitions: see main caption).

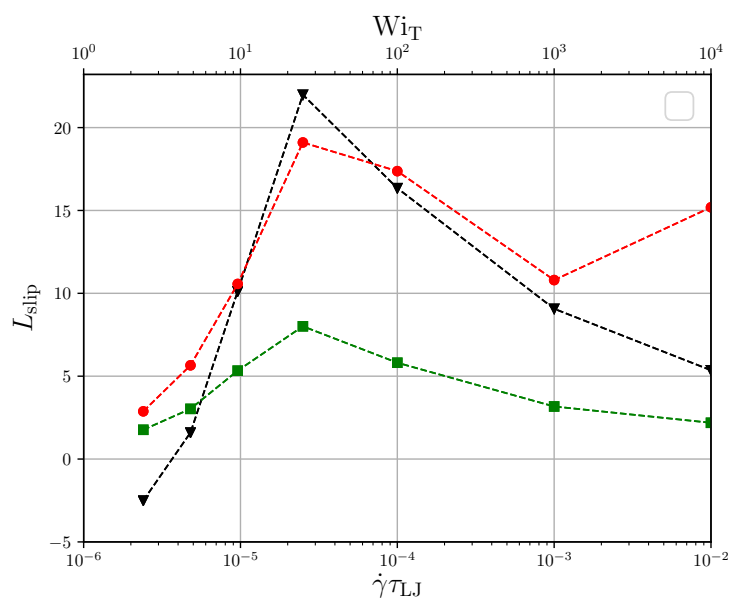

(b) Slip length (three definitions: see main caption). Note that the $y$ axis is not centered at 0 in this plot.

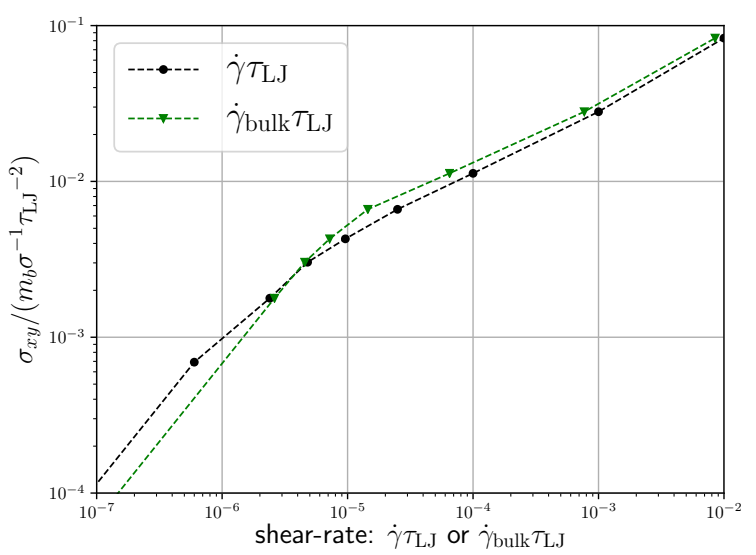

(d) Film flow curves using either the applied or bulk shear-rates.

FIG. 16. Various functions of applied shear-rate. (a) Ratio of bulk to applied shear-rate. (b) Slip length found in different ways. Red circles: $L_{\text {slip }}=V_{s}^{\mathrm{F}} / \dot{\gamma}_{\text {bulk }}$, where $V_{s}^{\mathrm{F}}$ is the mean value of the data points closest to the surface of the free monomer velocity profile, Fig. 6b Green squares: $L_{\text {slip }}=V_{s}^{\text {all }} / \dot{\gamma}_{\text {bulk }}$, where $V_{s}^{\text {all }}$ is the mean value of the data points closest to the surface of the total monomer velocity profile, Fig. 6b. Black triangles: slip length extrapolated from the center channel velocity profile - Eq. (6). (c) surface friction found from the relation Eq. (4), using the three different definitions of the slip length in Fig. $16 \mathrm{~b}$

effect has been postulated already [20]. In addition we identify a new mechanism that may also reduce the rheological significance of tethered-free kinks, resulting from the higher density of free chain end segments in the immediate proximity of the surface (Fig. 12b). Following the coil-stretch transition, the end segment excess correlates with an increased relative occupational density of free chain primitive path kinks, that are due to the proximity of a tethered primitive path, near contour ends (Fig. 14b). This may lead to a faster 
free chain constraint release rate acting on the tethered chains.

Different definitions of a dynamic surface friction, $\xi_{s}(\dot{\gamma})$, were investigated. In all cases the surface friction decreases most rapidly during the coil-stretch transition (see Fig. 16c), even though, as seen in Fig. 10, the actual drop in tethered-free kinks over this shear-rate range is quite low. However such kinks that are lost may be highly rheologically significant (for the reasons discussed above). The correlation between the number of tethered-free kinks and definitions of surface friction is more notable than the correlation between the number of kinks in the bulk and bulk viscosity.

A marginal regime in which the fluid stress does not increase with increasing applied shear-rate is not observed here. This might be due to the relatively weakly entangled chain length and the corresponding small separation in time-scales of surface disentanglement and bulk shear-thinning. However the same mechanisms leading to slip that we have identified here may remain relevant for polymer melts of much higher (and more experimentally relevant) molecular weights. The conclusions made here may therefore be used in the future to inform theoretical models for surface rheology.

\section{ACKNOWLEDGMENTS}

We thank Professor Jean-Louis Barrat for useful comments and advice. Dr. Dipesh Amin wrote a large amount of the GPU MD code and gave much useful advice regarding CUDA programming. Dr. Pawel Stasiak provided the simulation data using periodic boundaries and the SLLOD algorithm [57] that was included in Fig. 5a. This study was conceived of by Professor Alexei E. Likhtman.

[1] Morton. M. Denn, “Extrusion instabilities and wall slip," Annu. Rev. Fluid Mech. 33, 265 (2001).

[2] S. G. Hatzikiriakos, "Wall slip of molten polymers," Progr. Polym. Sci. 37, 624 (2012).

[3] S-Q. Wang and P. A. Drda, "Superfluid-like stick-slip transition in capillary flow of linear polyethylene melts. 1. General features," Macromolecules 29, 2627 (1996).

[4] J. L. Adams, S. M. Fielding, and P. D. Olmsted, "The interplay between boundary conditions and flow geometries in shear banding: Hysteresis, band configurations, and surface transitions," J. Non-Newtonian Fluid Mech. 151, 101 (2008).

[5] S. G. Hatzikiriakos, C. W. Stewart, and D. M. Dealy, "Effect of surface coatings on wall slip of LLDPE,” Intern. Polymer Processing VIII 8, 3033 (1993).

[6] S-Q. Wang and P. A. Drda, "Stick-slip transition in capillary flow of polyethylene .2. molecular weight dependence and low-temperature anomaly," Macromolecules 29, 4115 (1996). 
[7] S. M. Sabzevari, I. Cohen, and P. M. Wood-Adams, "Wall slip of bidisperse linear polymer melts," Macromolecules 47, 3154 (2014).

[8] J. K. Lee, B. Akgun, Z. Jiang, S. Narayanan, and M. D. Foster, "Altering surface fluctuations by blending tethered and untethered chains," Soft Matter 13, 8264-8270 (2017).

[9] Wei-Liang Chen, Roselynn Cordero, Hai Tran, and Christopher K Ober, "50th Anniversary Perspective: Polymer Brushes: Novel surfaces for future materials," Macromolecules 50, 4089 (2017).

[10] A. Chennevire, F. Cousin, F. Bou, E. Drockenmüller, K. R. Shull, L. Léger, and F. Restagno, "Direct molecular evidence of the origin of slip of polymer melts on grafted brushes," Macromolecules 49, 2348 (2016).

[11] N. C. Karayiannis and M. Kröger, "Combined molecular algorithms for the generation, equilibration and topological analysis of entangled polymers: Methodology and performance,” Int. J. Mol. Sci. 10, 5054-5089 (2009).

[12] Martin Kröger, "Shortest multiple disconnected path for the analysis of entanglements in two- and threedimensional polymeric systems." Comput. Phys. Commun. 168, 209 (2005).

[13] S. Shanbhag and M. Kröger, "Primitive path networks generated by annealing and geometrical methods: Insights into differences," Macromolecules 40, 2897 (2007).

[14] Jack Kirk, Zuowei Wang, and Patrick Ilg, "Entanglement dynamics at flat surfaces: investigations using multichain Molecular Dynamics and a single-chain slip-spring model," Under review.

[15] Ralf Everaers, "Topological versus rheological entanglement length in primitive-path analysis protocols, tube models, and slip-link models," Phys. Rev. E. 86, 022801 (2012).

[16] A.E. Likhtman, "The tube axis and entanglements in polymer melts," Soft Matter 10, 1895 (2014).

[17] Jing Cao, Jian Qin, and Scott T. Milner, “Simulating constraint release by watching a ring cross itself," Macromolecules 47, 2479 (2014).

[18] J. Cao and Z. Wang, "Microscopic picture of constraint release effects in entangled star polymer melts," Macromolecules 49, 5677 (2016).

[19] F. Brochard and P.-G. de Gennes, "Shear-dependent slippage at a polymer/solid interface," Langmuir 81, 3033 (1992).

[20] A. Ajdari, F. Brochard-Wyart, P.-G. de Gennes, and L. Leibler, "Slippage of an entangled polymer melt on a grafted surface," Physica A 204, 17-39 (1994).

[21] A. Ajdari, F. Brochard-Wyart, C. Gay, P.-G. de Gennes, and J. Viovy, "Drag on a tethered chain moving in a polymer melt,” J. Phys. II 5, 491 (1995).

[22] F. Brochard, C. Gay, and P.-G. de Gennes, "Slippage of polymer melts on grafted surfaces," Macromolecules 29, 377 (1996).

[23] K. B. Migler, H. Hervet, and L. Léger, "Slip transition of a polymer melt under shear stress," Phys. Rev. Lett. 70, 283 (1993).

[24] L. Léger, H. Hervet, G. Massey, and E. Durliat, "Wall slip in polymer melts," J. Phys: Cond. Mat. 9, 7719 (1997).

[25] S. T. Milner and T. C. B. Mcleish, "Reptation and contour-length fluctuations in melts of linear polymers," Phys. Rev. Lett. 81, 725 (1998). 
[26] V. Mhetar and L. A. Archer, "Slip in entangled polymer solutions," Macromolecules 31, 6639 (1998).

[27] P. E. Boukany and S-Q. Wang, "Exploring origins of interfacial yielding and wall slip in entangled linear melts during shear or after shear cessation,” Macromolecules 42, 2222 (2009).

[28] D. W. Mead and R. G. Larson, “A molecular theory for fast flows of entangled polymers," Macromolecules 31, 7895 (1998).

[29] S. T. Milner, T. C. B. McLeish, and A. E. Likhtman, "Microscopic theory of convective constraint release," J. Rheol. 45, 539 (2001).

[30] R. S. Graham, A. E. Likhtman, T. C. B. McLeish, and S. T. Milner, "Microscopic theory of linear, entangled polymer chains under rapid deformation including chain stretch and convective constraint release," J. Rheol. 47, 1171 (2003).

[31] Y. M. Joshi, A. K. Lele, and R. A. Mashelkar, "Molecular model for wall slip: Role of convective constraint release," Macromolecules 34, 3412 (2001).

[32] M. A. Tchesnokov, J. Molenaar, J. J. M. Slot, and R. Stepanyan, "A molecular model for cohesive slip at polymer melt/solid interfaces," J. Chem. Phys. 122, 214711 (2005).

[33] E. Durliat, H. Hervet, and L. Léger, "Influence of grafting density on wall slip of a polymer melt on a polymer brush,” Europhys. Lett. 38, 383 (1997).

[34] R. S. Hoy and G. S. Grest, "Entanglements of an end-grafted polymer brush in a polymeric matrix," Macromolecules 40, 8389 (2007).

[35] N. A. Rorrer and J. R. Dorgan, "Molecular-scale simulation of cross-flow migration in polymer melts," Phys. Rev. E. 90, 052603 (2014).

[36] J. R. Dorgan and N. A. Rorrer, "Molecular scale simulation of homopolymer wall slip," Phys. Rev. Lett. 110, 176001 (2013).

[37] X. Zhou, D. Andrienko, L. Delle Site, and K. Kremer, "Dynamic surface decoupling in a sheared polymer melt," Europhys. Lett. 70, 264 (2005).

[38] S. Jeong, S. Cho, J. M. Kim, and C. Baig, "Molecular mechanisms of interfacial slip for polymer melts under shear flow,” J. Rheol. 61, 253 (2017).

[39] A. Korolkovas, C. Rodriguez-Emmenegger, A. S. de los Pereira, A. Chennevire, F. Restagno, M. Wolff, F. A. Adlmann, A. J. C. Dennison, and P. Gutfreund, "Polymer brush collapse under shear flow," Macromolecules 50, 1215 (2017).

[40] M. Doi and S. F. Edwards, The Theory of Polymer Dynamics (Clarendon Press, 1986).

[41] J. Cao and A. E. Likhtman, "Time-dependent orientation coupling in equilibrium polymer melts." Phys. Rev. Lett. 104, 207801 (2010).

[42] M. Kröger, "Simple models for complex nonequilibrium fluids," Phys. Rep. 390, 453 (2004).

[43] K. Kremer and G. S. Grest, "Dynamics of entangled linear polymer melts: A molecular-dynamics simulation," J. Chem. Phys. 92, 5057 (1990).

[44] J. Kirk and P. Ilg, "Chain dynamics in polymer melts at flat surfaces," Macromolecules 50, 3703 (2017).

[45] J. Baschnagel and F. Varnik, "Computer simulations of supercooled polymer melts in the bulk and in confined 
geometry,” J. Phys.: Cond. Mat. 17, R851 (2005).

[46] B. C. Abberton, W. K. Liu, and S. Keten, "Anisotropy of shear relaxation in confined thin films of unentangled polymer melts," Macromolecules 48, 7631 (2015).

[47] M. P. Allen and D. J. Tildesley, Computer Simulation of Liquids (Oxford Science Publications, 1987).

[48] Jack Kirk, The molecular dynamics and rheology of polymer melts near the flat surface, Ph.D. thesis, School of Mathematical, Physical and Computational Sciences, University of Reading (2018).

[49] J. Cao and A. E. Likhtman, "Simulating startup shear of entangled polymer melts," ACS Macro. Lett. 4, 1376 (2015).

[50] A. E. Likhtman, "Viscoelasticity and molecular rheology," in Polymer Science: A Comprehensive Reference, Vol. 10, edited by K. Matyjaszewski and M. Müller (Elsevier B.V., 2012) p. 133.

[51] J. Sanders and E. Kandrot, CUDA by Example (Addison-Wesley, Upper Saddle River, NJ, 2010).

[52] D. Amin, Computational and theoretical modelling of self-healable polymer materials, Ph.D. thesis, School of Mathematical, Physical and Computational Sciences, University of Reading (2016).

[53] S. T. Milner, "Predicting the tube diameter in melts and solutions," Macromolecules 38, 4929 (2005).

[54] C. Baig, V. G. Mavrantzas, and M. Kröger, "Flow effects on melt structure and entanglement network of linear polymers: Results from a nonequilibrium molecular dynamics simulation study of a polyethylene melt in steady shear," Macromolecules 43, 6886 (2010).

[55] B. D. Todd, D. J. Evans, and P. J. Daivis, "Pressure tensor for inhomogeneous fluids," Phys. Rev. E 52, 16271638 (1995).

[56] M. Kröger and S. Hess, "Rheological evidence for a dynamical crossover in polymer melts via nonequilibrium molecular dynamics," Phys. Rev. Lett. 85, 1128 (2000).

[57] D. J. Evans and G. Morriss, Statistical mechanics of nonequilibrium liquids (Cambridge, 2008).

[58] R. S. Hoy, K. Foteinopoulou, and M. Kröger, "Topological analysis of polymeric melts: Chain length effects and fast-converging estimators for entanglement length,” Phys. Rev. E. 80, 031803 (2009).

[59] M. W. Matsen and P. Mahmoudi, "Segregation of chain ends to the surface of a polymer melt," Eur. Phys. J. E 37, 78 (2014).

[60] A. E. Likhtman, "Single-chain slip-link model of entangled polymers: Simultaneous description of neutron spinecho, rheology, and diffusion," Macromolecules 38, 6128 (2005).

[61] A. E. Likhtman and M. Ponmurugan, "Microscopic definition of polymer entanglements," Macromolecules 47, 1470 (2014).

[62] A. Ramirez-Hernandez, B. L. Peters, L. Schneider, M. Andreev, J. D. Schieber, M. Müller, M. Kröger, and J. J. de Pablo, "A detailed examination of the topological constraints of lamellae-forming block copolymers," Macromolecules 51, 2110-2124 (2018).

[63] N. V. Priezjev and S. M. Troian, "Molecular origin and dynamic behavior of slip in sheared polymer films." Phys. Rev. Lett. 92, 018302 (2004).

[64] M. Henot, A. Chennevire, E. Drockenmüller, L. Léger, and F. Restagno, "Comparison of the slip of a PDMS melt on weakly adsorbing surfaces measured by a new photobleaching-based technique," Macromolecules 50, 
5592-5598 (2017). 